\title{
CIRCUMSOLAR ENERGETIC PARTICLE DISTRIBUTION ON 2011 NOVEMBER 3
}

\author{
R. Gómez-Herrero ${ }^{1,2}$, N. Dresing ${ }^{3}$, A. Klassen ${ }^{3}$, B. Heber ${ }^{3}$, D. Lario ${ }^{4}$, N. Agueda ${ }^{5}$,

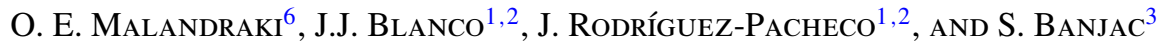 \\ ${ }^{1}$ SRG, Universidad de Alcalá, E-28871 Alcalá de Henares, Spain; raul.gomezh@uah.es \\ ${ }^{2}$ Guadalab, Parque Científico y Tecnológico de Guadalajara, E-19005 Guadalajara, Spain \\ ${ }^{3}$ IEAP, Christian-Albrechts-Universität zu Kiel, D-24118 Kiel, Germany \\ ${ }^{4}$ The Johns Hopkins University, Applied Physics Laboratory, Laurel, MD 20723, USA \\ ${ }^{5}$ Departament d'Astronomia i Meteorologia. Institut de Ciències del Cosmos. Universitat de Barcelona, E-08028 Barcelona, Spain \\ ${ }^{6}$ IAASARS, National Observatory of Athens, GR-15236 Penteli, Greece \\ Received 2014 June 19; accepted 2014 November 5; published 2015 January 15
}

\begin{abstract}
Late on 2011 November 3, STEREO-A, STEREO-B, MESSENGER, and near-Earth spacecraft observed an energetic particle flux enhancement. Based on the analysis of in situ plasma and particle observations, their correlation with remote sensing observations, and an interplanetary transport model, we conclude that the particle increases observed at multiple locations had a common single-source active region and the energetic particles filled a very broad region around the Sun. The active region was located at the solar backside (as seen from Earth) and was the source of a large flare, a fast and wide coronal mass ejection, and an EIT wave, accompanied by type II and type III radio emission. In contrast to previous solar energetic particle events showing broad longitudinal spread, this event showed clear particle anisotropies at three widely separated observation points at $1 \mathrm{AU}$, suggesting direct particle injection close to the magnetic footpoint of each spacecraft, lasting for several hours. We discuss these observations and the possible scenarios explaining the extremely broad particle spread for this event.
\end{abstract}

Key words: Sun: activity - Sun: coronal mass ejections (CMEs) - Sun: flares - Sun: heliosphere Sun: particle emission

\section{INTRODUCTION}

Solar energetic particle (SEP) events are commonly classified into two distinct classes: impulsive and gradual (e.g., Reames 1988, 1999, 2013). Impulsive SEP events are believed to be the consequence of particle acceleration in a spatially compact region, where magnetic reconnection takes place during solar flares. This kind of events show relatively short temporal profiles, are electron-rich, and tend to show, among other compositional signatures, large enhancements of the ${ }^{3} \mathrm{He} /{ }^{4} \mathrm{He}$ isotopic ratio with respect to the coronal and solar wind abundances. Gradual SEP events are the result of particle acceleration in the corona and the solar wind by shock waves driven by coronal mass ejections (CMEs). They are characterized by longer time durations and typically by much higher intensities than impulsive events, and their composition is closer to the coronal and solar wind abundances. According to this picture, impulsive events generate narrow particle distributions, only observable when the observer's magnetic footpoint is within $\sim 20^{\circ}$ from the source region (Reames 1999), while gradual events are able to produce much wider longitudinal distributions due to the extended nature of the source. During gradual events, the shape of the particle intensity temporal profiles is conditioned by the dynamic evolution of the shock strength and by the location of the observer with respect to the traveling CME-driven shock (e.g., Cane et al. 1988).

Observations carried out by single spacecraft orbiting near Earth have shown the existence of SEP events with sources located at longitudes well separated from the nominally magnetic well-connected regions. For example, there have been cases of SEP events originating at longitudes close to $180^{\circ}$ apart from the Central Meridian line (Dodson \& Hedeman 1969; Torsti et al. 1999; Cliver et al. 2005) or at longitudes close to the east limb (Cliver et al. 1995). Such gradual events have been often interpreted in terms of acceleration at CME-driven coronal shocks covering broad angular regions, meaning that the most extreme propagation cases could provide an indirect evidence of the existence of "quasi-circumsolar" shocks (Cliver et al. 1995).

A primary tool to investigate the spatial distribution of energetic particles during SEP events is the combination of observations performed simultaneously by spacecraft at different locations in the heliosphere. Out-of-the-ecliptic observations by Ulysses have proven that solar energetic particles often reach high heliographic latitudes (e.g., Dalla et al. 2003b, 2003a; Lario et al. 2003; Malandraki et al. 2009; McKibben et al. 2003). The delay in the particle onset observed at high latitudes compared with in-ecliptic observations has been interpreted in terms of either interplanetary (IP) cross-field diffusion or as a consequence of the time required by a shock to intercept the magnetic field lines connecting to the high-latitude observer (e.g., Malandraki et al. 2009). Similar multi-point observations of SEP events widely separated in longitude have been performed in the ecliptic plane combining Helios and near-Earth observatories (e.g., Kallenrode 1993b, 1993a; Lario et al. 2006). Wibberenz \& Cane (2006) analyzed observations of impulsive events by the two Helios and near-Earth spacecraft and found that relativistic electrons are often detectable at longitudinal separations beyond $80^{\circ}$ from the flare position.

The Solar-TErrestrial RElations Observatory (STEREO; Kaiser 2005) consists of two nearly identical spacecraft launched in 2006 October. Both spacecraft follow heliocentric orbits, with STEREO-A moving around the Sun faster than Earth and STEREO-B moving slower. This results in progressively increasing longitudinal separations with respect to Earth while both STEREO spacecraft stay always at heliocentric distances close to $1 \mathrm{AU}$ and near the ecliptic plane, minimizing the effect of radial and latitudinal energetic particle gradients. This configuration provides an ideal platform to study the longitudinal variations of SEP event properties near $1 \mathrm{AU}$ (see, e.g., Dresing et al. 2012, 2014; Cohen et al. 2012; Lario et al. 2013; Mewaldt 
et al. 2013; Richardson et al. 2014). In contrast to Ulysses and Helios, apart from the in situ instrumentation, both STEREO spacecraft are equipped with a complete set of remote-sensing instruments, which continuously monitor the solar surface and track the propagation of solar disturbances from the corona to the IP medium. Wiedenbeck et al. (2013) reported combined near-Earth and STEREO observations showing that the detection of ${ }^{3} \mathrm{He}$-rich events from a pair of spacecraft (s/c) separated by more than $60^{\circ}$ is not uncommon, and in some cases, such as the 2010 February 7 event, ${ }^{3} \mathrm{He}$ increases become observable over regions covering more than $130^{\circ}$ in longitude at $1 \mathrm{AU}$. Dresing et al. (2012) used STEREO and near-Earth observations to investigate the longitudinal distribution of energetic particles for the 2010 January 17 SEP event. During this event, energetic electrons and protons were observed over a wide longitudinal range at $1 \mathrm{AU}$, while the magnetic footpoints of the three $\mathrm{s} / \mathrm{c}$ were separated by more than $100^{\circ}$ in longitude from the source active region (AR). This event onsets showed large delays and lack of clear anisotropies. The event was accompanied by type II radio emission and by an EIT wave, but there were no observational evidences of a particularly wide shock able to reach the magnetic footpoints of the three spacecraft. Using a threedimensional SEP propagation model, the authors concluded that the main observational features of the event and the nearly $360^{\circ}$ particle spread can be explained in terms of SEP perpendicular diffusion in the IP medium.

Observations of gradual events have been successfully reproduced using numerical models that combine particle injection at an evolving CME-driven shock moving away from the Sun and energetic particle transport throughout the interplanetary medium (e.g., Heras et al. 1992, 1995; Kallenrode \& Wibberenz 1997; Lario et al. 1998; Lee 2005; Zank et al. 2007; Luhmann et al. 2007, 2010; Verkhoglyadova et al. 2009). The complexity of these models has increased in order to account for contributions such as self-amplified waves (e.g., Lee 2005; $\mathrm{Ng}$ et al. 2003), shock acceleration processes (e.g., Zank et al. 2000; Li et al. 2003; Li et al. 2005), the presence of multiple shocks, or the possible mixture of a flare-accelerated component which can be further accelerated at the shock (Li \& Zank 2005; Li et al. 2012; Verkhoglyadova et al. 2010). These models also have important applications from the point of view of space weather forecasting (e.g., Aran et al. 2008; Luhmann et al. 2010; Verkhoglyadova et al. 2012; Horne et al. 2013, and references therein).

\subsection{Physical Processes Responsible for the Longitudinal Spread of SEPS}

The observations described above illustrate the observational evidence of SEP distributions covering wide angular regions at $1 \mathrm{AU}$. Several physical processes have been proposed in the literature to explain how energetic particles are distributed in longitude either close to the Sun or during IP transport.

1. An extended or moving particle source. Acceleration at coronal or IP shocks covering or propagating over extreme longitudinal distances of up to $\sim 300^{\circ}$ would provide a straightforward explanation for wide-spread SEP events. Indeed, observations of SEP originating from active regions poorly connected to the observer have been often interpreted as an indirect evidence of very wide coronal shocks (e.g., Cliver et al. 1995, 2005). However, direct observational evidence of such wide shocks has not been found. Based on a sample of 40 IP shocks, Richardson \& Cane (1993) concluded that shock drivers (understood as the ejecta or the interplanetary counterpart of the CME, henceforth ICME) can extend at $1 \mathrm{AU}$ up to a full-width of about $100^{\circ}$ in longitude, centered on the solar source longitude. The IP shocks themselves extend beyond the longitudinal span of the corresponding ICME (Cane 1988, 1995), but, according to Cane (1996), the longitudinal extent of IP shocks at $1 \mathrm{AU}$ is at most $180^{\circ}$. Using multi-point observations by Helios, IMP-8, and ISEE-3, de Lucas et al. (2011) investigated the angular extent of ICME-driven IP shocks during 1974-1986. They concluded that shock waves have about a $50 \%$ chance to be observed by two different locations separated by $90^{\circ}$. The number of multi-spacecraft observed shocks clearly drops off for separation angles larger than $100^{\circ}$, and only four cases were found covering angular separations between $120^{\circ}$ and $160^{\circ}$.

While the angular extent of IP shocks can be directly investigated using multi-point in situ measurements, the size of coronal shocks can only be indirectly inferred via remote-sensing observations of the electromagnetic emissions associated with them. According to Nelson \& Robinson (1975), the average angle subtended at the solar surface by fundamental metric type II radio emission sources is $43^{\circ}$. Aurass et al. (1994) found particular cases with larger, double type II source structures covering a separation angle beyond $90^{\circ}$. Type II radio sources often show non-radial propagation trajectories (see Mann et al. 2003, and references therein). Wave-like large-scale disturbances propagating over the solar disk in extreme ultraviolet observations (usually referred to as "EIT waves" or "EUV waves") are in close empirical correlation with type II radio bursts (Klassen et al. 2000). Most EIT waves are accompanied by CMEs, and observations and MHD modeling suggest that they are driven by the lateral expansion of CMEs, while the ultimate nature of the phenomenon remains under debate and could consist of true waves, pseudo waves (e.g., reconnection fronts), or a combination of both (Patsourakos \& Vourlidas 2012; Nitta et al. 2013b, and references therein). According to Patsourakos \& Vourlidas (2012), EIT waves can reach distances up to $1.3 R_{\odot}(850 \mathrm{Mm})$ from the source. Singlecase studies reported some EIT waves covering a whole solar hemisphere (Klassen et al. 2000; Kienreich et al. 2009; Thompson \& Myers 2009). Connections between EIT waves and SEP events have been often suggested (e.g., Bothmer et al. 1997; Krucker et al. 1999), and recently Rouillard et al. (2012) hypothesized that the EIT wave can be used to track the expansion of a coronal shock responsible for particle acceleration. Other authors question the EIT wave acceleration scenario for SEPs, with many EIT waves being observed at well-connected positions having no associated SEP increase (Miteva et al. 2014).

2. Longitudinal particle transport in the corona. The existence of some kind of "coronal diffusion" process was one of the earliest suggested hypotheses to explain fast SEP propagation far from the optimal connected longitudes during impulsive SEP events (Reid 1964). Different physical processes were proposed to be responsible for such diffusion, for instance, field line reconnection allowing the transfer of particles between neighbor coronal loops (Newkirk \& Wentzel 1978). Reinhard \& Wibberenz (1974) examined the time of SEP maximum intensity versus the source longitude, concluding that there is a wide region extending up to $50^{\circ}$ in longitude from the AR, where the particles 
propagate equally fast (the so-called Fast Propagation Region (FPR)), suggesting the existence of a fast azimuthal propagation process operating close to the Sun. Field lines emerging from active regions often show a strongly diverging topology between the photosphere and the solar wind source surface, as illustrated by Potential Field Source Surface (PFSS) models (e.g., Schrijver \& De Rosa 2003). Klein et al. (2008) used PFSS models and radio-heliographic observations to trace the coronal propagation of energetic particles, concluding that particles can propagate along open flux tubes rapidly diverging with height, connecting the parent active region to the Parker spiral at the source surface even when the active region is as far as $50^{\circ}$ away from the nominal connection longitude. A new model presented by Masson et al. (2012) proposed that energetic particles can be injected continuously along open field lines that are undergoing slip-running reconnection. Field lines could slip over large distances progressively farther from the acceleration site.

3. Sympathetic activity. Sometimes SEP events are nearly simultaneously observed by widely separated spacecraft, although they are originating from different active regions. This can be either the result of unrelated solar activity at distant active regions that (by chance) are closely spaced in time, or consequence of a physical link through largescale coronal magnetic field (see, e.g., Wang et al. 2001; Schrijver \& Title 2011; Shen et al. 2012; Schrijver et al. 2013).

4. Cross-field transport in the IP medium. Energetic charged particles traveling through the interplanetary medium can effectively propagate perpendicular to the average direction of the interplanetary magnetic field (Dröge et al. 2010; Giacalone \& Jokipii 2012, and references therein). There are several physical processes contributing to the particle movement across the field: drifts, scattering by interplanetary magnetic field turbulence, field line meandering, or interaction with magnetic field decreases (da Costa et al. 2013). Drift effects are commonly assumed to be negligible for SEP propagation; however, recent simulation works by Marsh et al. (2013) and Dalla et al. (2013) suggest that drifts due to curvature and gradient of Parker spiral could be a considerable source of asymmetric cross field transport for high-energy SEPs. The existence of particle intensity dropouts (Mazur et al. 2000) suggests that there is little diffusion across magnetic field lines during SEP propagation, consistent with very low values of the perpendicular to parallel diffusion coefficient ratio $\kappa_{\perp} / \kappa_{\|} \sim 10^{-4}$. Even in the absence of cross-field diffusion of the particles from the initial field lines, largescale field line meandering can be an effective contribution to cross-field transport (Giacalone \& Jokipii 2012; Kelly et al. 2012; Laitinen et al. 2013). According to Giacalone \& Jokipii (2012), for SEPs below $1 \mathrm{GeV}$, crossfield transport results primarily from the particles following meandering magnetic field lines (see also Giacalone \& Jokipii 1999). Observations of Jovian electrons propagating from Jupiter to Earth and modulation of low-energy galactic cosmic rays suggest values of $\kappa_{\perp} / \kappa_{\|} \sim 10^{-2}$ (Chenette 1980; Ferreira et al. 2001; Heber \& Potgieter 2006). Based on the analysis of time delays between type III radio burst reaching the local plasma frequency and the burst onset at the Sun, and its comparison with SEP arrival time delays, Cane \& Erickson (2003) concluded that SEP cross-field transport occurs in the interplanetary medium. Interplanetary transport models including crossfield diffusion have been used by different authors to infer the variations of particle anisotropies, intensities, and time delays as a function of the source longitude in SEP events (e.g., Dröge et al. 2010; Wang et al. 2012). Simulations by Dresing et al. (2012) required values of the perpendicular to parallel mean-free path ratio as large as $\sim 0.3$ in order to reproduce the observations of the wide-spread nearly relativistic electron event on 2010 January 17. Variable conditions in the interplanetary magnetic field turbulence provide a natural source of variability of $\kappa_{\perp} / \kappa_{\|}$values, which would explain why SEP behavior varies between very broad longitudinal spread events and spike-like events that require nearly scatter-free propagation (e.g., Klassen et al. 2011, 2012). Since the Parker spiral geometry is not azimuthally symmetric, West-East asymmetries can arise as a result of the IP particle transport (He et al. 2011).

5. Deviations from the ideal interplanetary magnetic field spiral structure. Large-scale transient structures propagating through the solar wind such as ICMEs can substantially modify the structure of the interplanetary magnetic field. An observer crossing these structures can become connected to a different location in the corona, shifted from the nominal connection point (e.g., Richardson et al. 1991; Richardson \& Cane 1996; Torsti et al. 2004; Chollet et al. 2010). Even if the ICME is not passing by the observer's location, it can distort the Parker spiral between the observer and the Sun, displacing the nominal location of the magnetic footpoint (e.g., Gómez-Herrero et al. 2007). These kinds of processes can provide an explanation for SEP events originating from active regions distant from the optimal nominal connection, while undisturbed lines could still transport particles to a different observer well connected to the source region.

Complex scenarios involving a combination of more than one of the processes described above are likely to happen (Dresing et al. 2014). For instance, cross-field diffusion combined with acceleration by a moving IP shock provides a scenario in which an observer could detect particles before being magnetically connected to the shock (Wang et al. 2012). Single observational signatures can have multiple interpretations: for instance, time delays of particle onsets can be interpreted in terms of (1) the time needed by a shock to laterally intercept a field line connecting to the observer, (2) the time required for the shock to reach certain height in the corona (Kahler 1994; Reames 2009a), or (3) as diffusion times in the IP medium required for the particles to reach the field line connected to the observer. Moreover, often these times are comparable, and therefore it is difficult to discern the processes responsible for the delay in the first arriving particles. Consequently, timing alone does not provide enough information to firmly establish the physical scenario under which particles reached the field lines connected to the observer. Additional insights can be obtained analyzing other observational signatures such as particle anisotropies that are of critical importance to determine if the source directly injects particles in a field line connecting with the observer (e.g., Agueda et al. 2014).

\subsection{Circumsolar Energetic Particle Increase on 2011 November 3}

Late on 2011 November 3, Earth was separated by 105.3 of heliographic longitude with respect to STEREO-A and by 102.5 


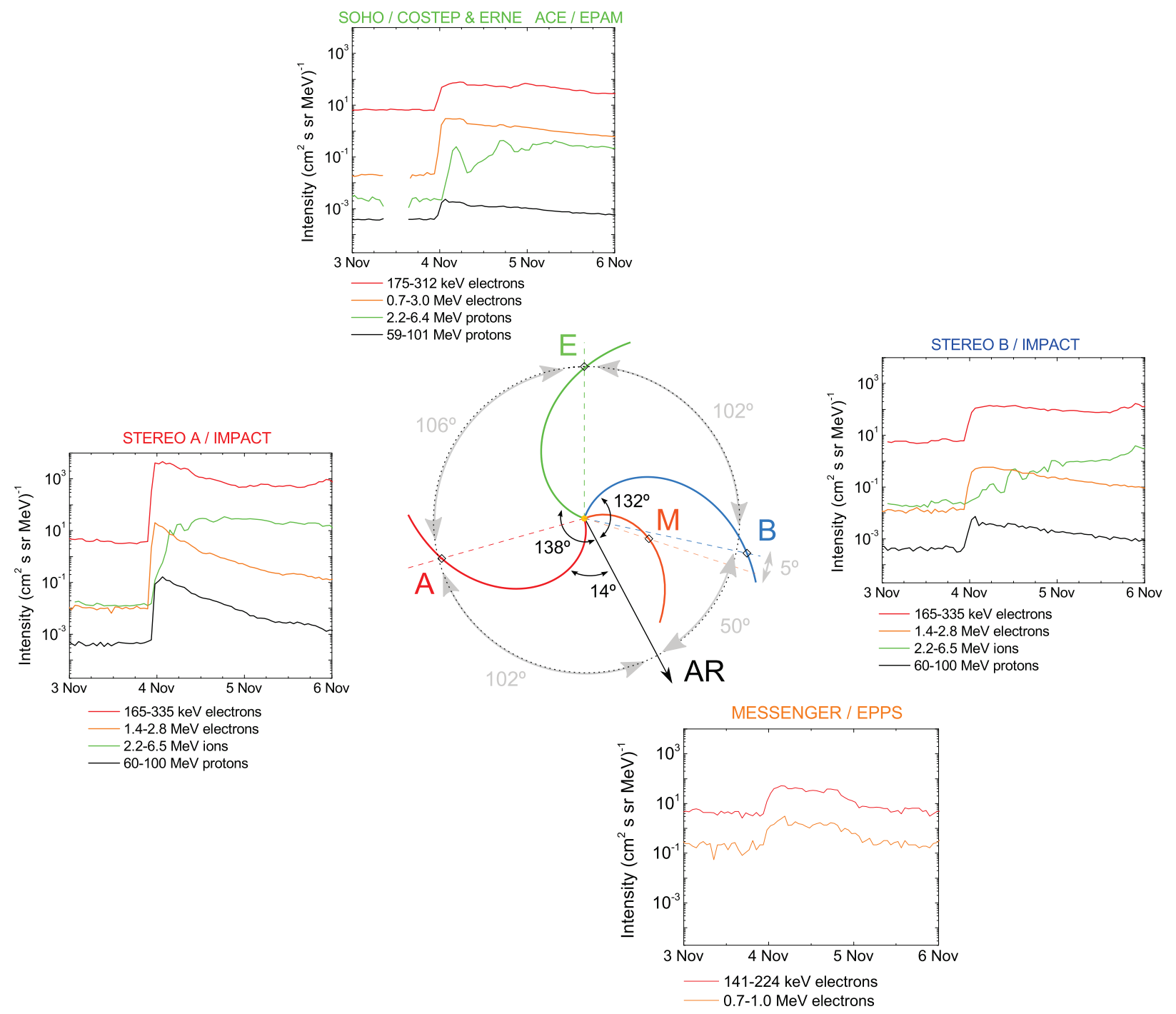

Figure 1. Overview of the 2011 November 3 nearly circumsolar energetic particle enhancement: electron and proton increases observed by STEREO-B, STEREO-A, $S O H O, A C E$, and MESSENGER. The central panel shows the orbital location of each spacecraft and nominal Parker spirals connecting them with the Sun. These spirals correspond to the solar wind speed observed in situ at the time of particle onset. The black arrow shows the location of the active region that is the likely source of the event, located $102^{\circ}$ west from STEREO-A 's point of view. The inner black arches mark the longitudinal separation between the spacecraft magnetic footpoints and the active region.

with respect to STEREO-B, with the longitudinal separation between both STEREO spacecraft being 152 .2. At the end of the day, energetic particle increases were observed by in situ particle instruments on board both STEREO spacecraft as well as by Wind, the Advanced Composition Explorer (ACE), and the Solar and Heliospheric Observatory (SOHO) orbiting near Earth. The increase was also observed by the MErcury Surface, Space ENvironment, GEochemistry, and Ranging (MESSENGER) spacecraft, in orbit around Mercury at 0.44 AU from the Sun and $107^{\circ}$ eastward from Earth ( $5^{\circ}$ eastward from STEREO-B). Figure 1 shows a sketch of the orbital location of STEREO-A, STEREO-B, Earth, and MESSENGER, the nominal Parker spirals connecting them with the Sun, and a summary of the energetic electron and proton observations at different energy ranges. Clear enhancements of proton intensities up to energies above $60 \mathrm{MeV}$ and relativistic electron intensities beyond $2 \mathrm{MeV}$ were observed near Earth and by both STEREO spacecraft. MES$S E N G E R$ does not provide high-energy proton observations, but a clear intensity increase was observed by all the electron channels, covering the $20-1000 \mathrm{keV}$ energy range. Relativistic electron increases started at 22:38 UT $\pm 3 \mathrm{~min}$ at STEREO-A,
23:35 UT $\pm 10 \mathrm{~min}$ at STEREO-B, at 23:02 $\pm 2 \mathrm{~min}$ at $S O H O$, and at 23:07 $\pm 5 \mathrm{~min}$ at MESSENGER (see Section 4.1 for a caveat on $S O H O$ and MESSENGER pointing directions).

This event has been analyzed by Park et al. (2013) and Prise et al. (2014), with deeper focus on remote-sensing observations. The analysis by Prise et al. (2014) suggested that the particle intensity enhancement observed by both STEREO spacecraft had a common origin, while the increase observed by Wind (located near Earth) probably originated from a different solar event. Conversely, Park et al. (2013) suggested different origins for the SEP events observed by STEREO-A and $S T E R E O-B$ and a common origin for the energetic protons observed by STEREO-A and Earth. This event is also included in the analysis of more than 200 proton events observed by STEREO recently presented by Richardson et al. (2014), who considered that the increases observed from these three locations had a common solar source rather than being being the result of multiple injections by sympathetic flares. The goal of this paper is to present observational evidences confirming a common solar origin for this almost circumsolar particle enhancement (CPE) and the discussion of the possible 
physical scenarios responsible for the broad particle spread around the Sun.

\section{INSTRUMENTATION}

We use data from instruments on board STEREO-A, STEREO-B, ACE, SOHO, Wind, the Geostationary Operational Environmental Satellites (GOES), MESSENGER and the Solar Dynamics Observatory ( $S D O$ ). Remote-sensing observations of the CME and the activity phenomena over the solar surface were provided by the Sun Earth Connection Coronal and Heliospheric Investigation (SECCHI) instrument suite on board STEREO (Howard et al. 2008), the Large Angle and Spectrometric Coronagraph experiment (LASCO) on board SOHO (Brueckner et al. 1995), the Atmospheric Imaging Assembly (AIA; Lemen et al. 2012) on board SDO, and the X-ray telescopes on board GOES. Synoptic maps including PFSS model results provided by the Global Oscillation Network Group (GONG, http://gong.nso.edu/) and by Solarsoft PFSS package were also examined. Radio observations were provided by the S/WAVES (Bougeret et al. 2008) investigation on board STEREO and by the WAVES (Bougeret et al. 1995) experiment on board Wind. In situ energetic particle observations were provided by the Solar Electron and Proton Telescope (SEPT; Müller-Mellin et al. 2008), the Low Energy Telescope (LET; Mewaldt et al. 2008), and the High Energy Telescope (HET; von Rosenvinge et al. 2008) on board STEREO (all of them part of the IMPACT instrument suite; Luhmann et al. 2008), the Comprehensive Suprathermal and Energetic Particle Analyzer (COSTEP; Müller-Mellin et al. 1995), and the Energetic and Relativistic Nuclei and Electron (ERNE; Torsti et al. 1995) instrument on board SOHO, the Electron, Proton, and Alpha Monitor (EPAM; Gold et al. 1998) on board $A C E$, the 3D Plasma and Energetic Particle Investigation (3DP) on board Wind, and the Energetic Particle and Plasma Spectrometer (EPPS; Andrews et al. 2007) on board MESSEN$G E R$. Additionally, $\mathrm{Fe} / \mathrm{O}$ ratios at $A C E$ were obtained using data from the Solar Isotope Spectrometer (SIS; Stone et al. 1998). Finally, solar wind plasma and magnetic field data were obtained from the Plasma and Suprathermal Ion Composition (PLASTIC; Galvin et al. 2008) investigation on board STEREO, the STEREO Magnetic Field Experiment (Acuña et al. 2008), the $A C E$ Magnetic Field Experiment (Smith et al. 1998), and the $A C E /$ Solar Wind Electron Proton Alpha Monitor (SWEPAM; McComas et al. 1998). Due to gaps in ACE/SWEPAM data, plasma data from the Charge, Element, and Isotope Analysis System (CELIAS; Hovestadt et al. 1995) instrument on board $S O H O$ were also used. Magnetic field data from MESSENGER were obtained from the Magnetometer Instrument on board this s/c (Anderson et al. 2007). Interplanetary disturbance identifications were cross-checked using the STEREO and ACE level 3 event list maintained by L. Jian (http://www-ssc.igpp.ucla.edu/ forms/stereo/stereo_level_3.html, http://www.srl.caltech.edu/ ACE/ASC/DATA/level3/) and the near-Earth ICME list maintained by I. Richardson and H. Cane (http://www.srl.caltech.edu/ ACE/ASC/DATA/level3/icmetable2.htm; see also Cane \& Richardson 2003).

\section{OBSERVATIONS}

\subsection{Remote-sensing Observations}

The top panel of Figure 2 shows the soft X-ray flux measured by GOES-15 from November 3 21:00 to November 4 00:00 UT. GOES observed four C-class X-ray flares starting at 22:12 UT, 22:28 UT, 22:56 UT, and 23:05 UT, and one M-class flare starting at 23:27 UT. The GOES X-ray flare list maintained

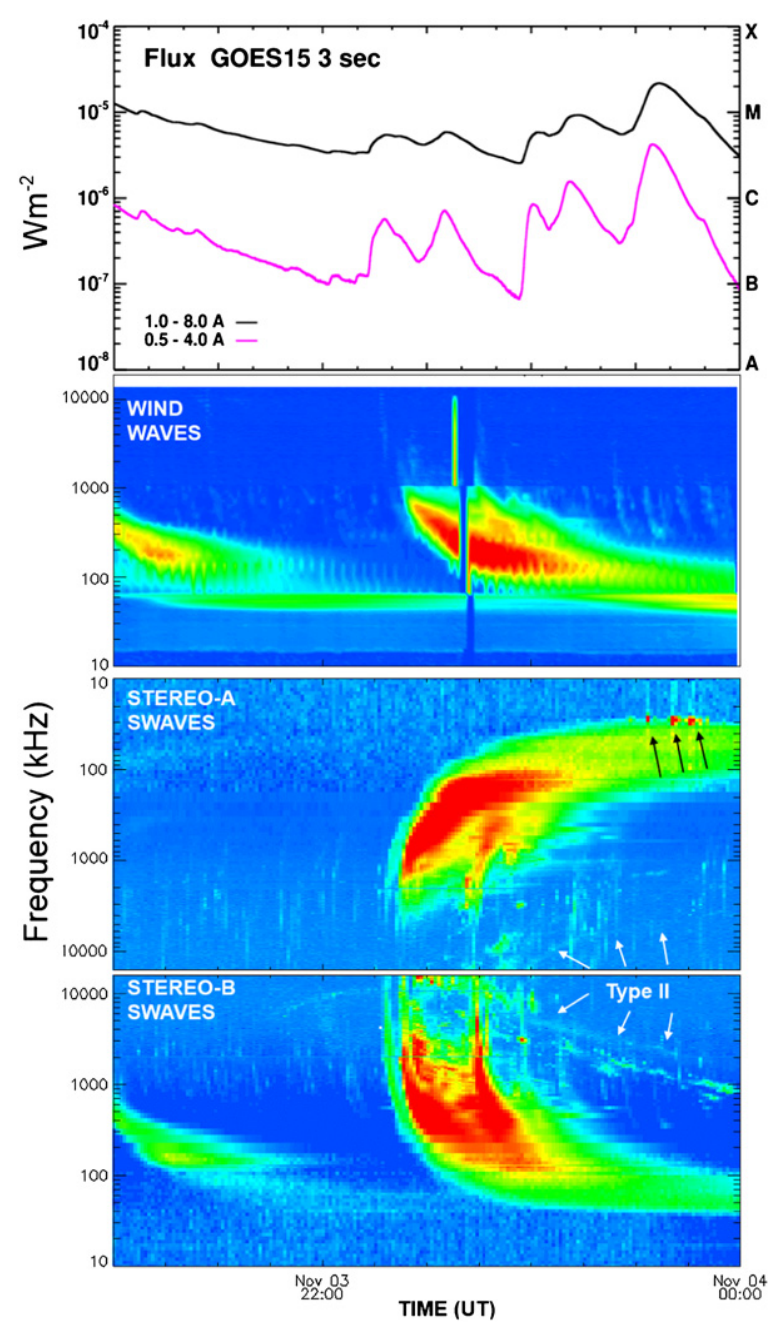

Figure 2. Top panel: soft X-ray observations by GOES 15 between November 3, 21:00 UT and November 4, 00:00 UT. Panels 2-4: radio dynamic spectra observed by Wind/WAVES, STEREO-A/SWAVES, and STEREO-B/SWAVES during the same time interval. The sharp intensity decrease observed by Wind/ WAVES at $\sim 1 \mathrm{MHz}$ is caused by the different sensitivity of the RAD1 and RAD2 receivers. At higher frequencies, a genuine attenuation was observed by $\mathrm{RAD} 2$. A type II radio burst observed by STEREO-A and STEREO-B is marked by white arrows. The black arrows mark a low-frequency intensification of the type III radio emission observed by STEREO-A, indication of locally generated Langmuir waves (see text for further details).

by NOAA/NGDC attributes all these soft X-ray increases to NOAA AR\#11339, located at N20E62 from Earth's point of view, $40^{\circ}$ eastward of the nominal magnetic footpoint of STEREO-B. According to Park et al. (2013), the flare at 22:12 UT originated from NOAA AR\#11333, close to the solar west limb, but the examination of AIA images supports the source location at AR\#11339. This region showed considerable activity during the day, including a large X1.9 flare at 20:16 UT (not shown in Figure 2), which in spite of the large X-ray intensity was radio-silent (Park et al. 2013). According to Chen et al. (2013), this X1.9 flare was accompanied by a failed filament eruption that remained confined by pre-existing magnetic arcades.

Panels 2-4 in Figure 2 show the radio dynamic spectra measured by Wind/WAVES, STEREO-A/SWAVES, and STEREO-B/SWAVES, respectively. The three spacecraft observed a sequence of type III radio bursts starting at 22:17 UT (determined at a frequency of $1 \mathrm{MHz}$ ), showing a strong attenuation above $4 \mathrm{MHz}$ for STEREO-A and Wind. For 
STEREO-B
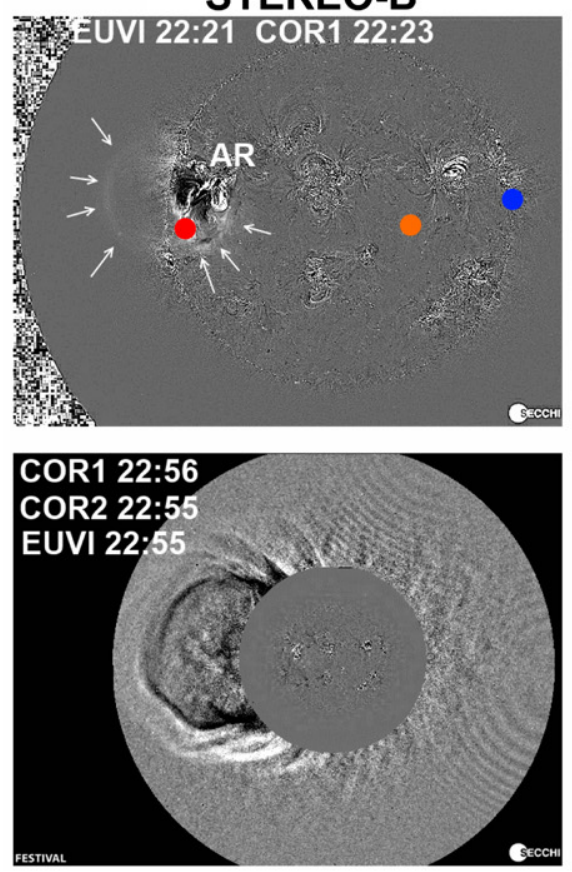

SDO / SOHO
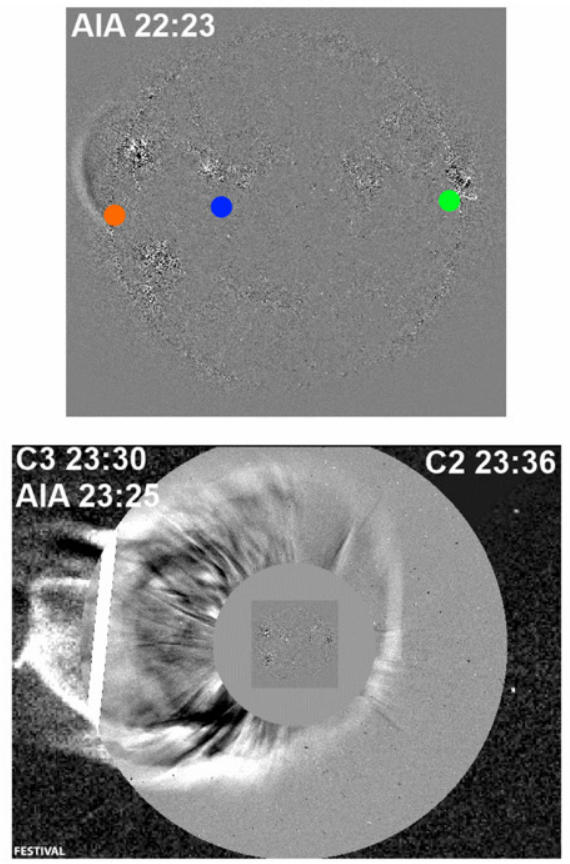

STEREO-A
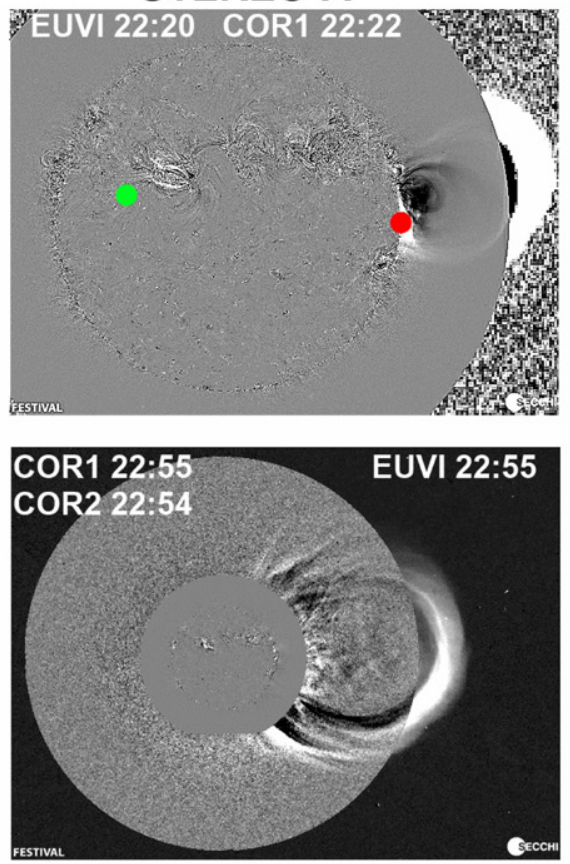

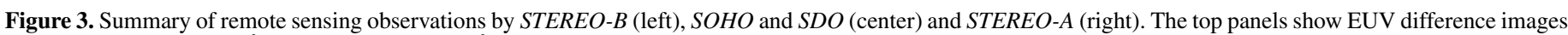

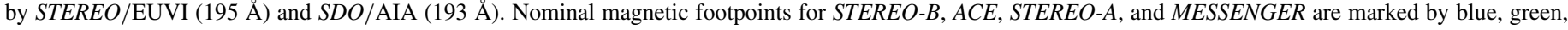

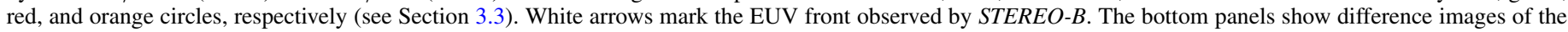
CME observed by SECCHI and LASCO coronographs.

STEREO-B this sequence was more intense and did not show high-frequency attenuation. STEREO-A observed a number of bursty intensifications at the low boundary of the type III radio emission close to $25 \mathrm{kHz}$, when it reached the local plasma frequency (marked by black arrows in the figure). This signature is commonly interpreted as an indication of Langmuir waves locally generated when the electron beam responsible for the type III radio burst reaches a spacecraft magnetically connected to the source flaring region (e.g., Lin 1990; Ergun et al. 1998; Cane 2003; Klassen et al. 2012; Thejappa et al. 2012). STEREO-B observed a clear type II radio burst (see white arrows in the figure), starting at the upper limit of SWAVES frequency range $(16 \mathrm{MHz})$ at around 22:30 UT, overlapped with the type III burst sequence. This type II radio burst showed fundamental and harmonic emission lanes, slowly drifting with intermittent emission to low frequencies, being still visible below $1 \mathrm{MHz}$ early on November 4 (not shown in the figure). The type II burst was also visible at STEREO-A, but with weaker intensity in comparison to STEREO-B. There were no clear evidences of type II radio emission at Wind (see http://ssed.gsfc.nasa.gov/waves/bursts_2011.html).

Since the solar backside remained invisible for GOES, the STEREO-SECCHI extreme ultraviolet imager (EUVI) on board both STEREO spacecraft can be used for flare identification (Nitta et al. 2013a). A summary of EUV and coronographic observations from STEREO-B, SOHO/SDO, and STEREO-A is shown in Figure 3. Nominal magnetic footpoints along the Parker spiral for STEREO-B, ACE, STEREO-A, and MESSEN$G E R$ are marked in the top panels by blue, green, red, and orange solid circles, respectively. These footpoints have been estimated using the one-hour averaged solar wind speed measured in situ at the time of electron onset at STEREO-B, ACE, and STEREO-A and a fixed value of $350 \mathrm{~km} \mathrm{~s}^{-1}$ for MESSENGER (see Section 3.3). The nominal magnetic footpoint of STEREO-A was located at less than $15^{\circ}$ eastward from the AR; whereas the sepa- rations of the nominal footpoints of MESSENGER, STEREO-B, and Earth with respect to the AR were $75^{\circ}, 132^{\circ}$, and $138^{\circ}$, respectively.

STEREO-B/EUVI observed a large two-ribbon flare at $\mathrm{N} 10 \mathrm{E} 50$ from that s/c point of view, which corresponds to $12^{\circ}$ behind the west limb from the point of view of STEREO-A and to the distant backside (E152) from Earth's point of view. Evolving loops were observed above this AR before 22:00 UT, and the loop brightening started between 22:11 and 22:16 UT at the top of the loop, southwest from the AR. According to Nitta et al. (2013a), who developed a method to estimate soft X-ray flare peak intensities from EUV $195 \AA$ fluxes, the $\mathrm{X}$-ray flare peaked at 22:41 UT, with an estimated peak intensity between M4.7 and X1.4 (henceforth the M4.7-X1.4 flare). An EIT wave propagating from this flaring AR was clearly observed in STEREO-B/EUVI difference images. By 22:21 UT, this EIT wave had already surpassed STEREO-A magnetic footpoint (see top left panel in Figure 3 ). The wave disappeared around central heliolongitudes (from the STEREO-B point of view), before reaching the $S T E R E O-B$ nominal magnetic footpoint. Figure 4 shows $S T E R E O / E U V I$ and $S D O /$ AIA difference images at $\sim 22: 21 \mathrm{UT}$. The contrast has been enhanced to highlight the expanding "dome" structure observed over the solar limb by the three s/c. The approximate geometry of this structure is sketched in the center of the figure. Note that this structure is not spherically symmetric around the flare location (marked by a black arrow), but a slight shift toward STEREO-B is required to reproduce the observations. From the $S D O$ point of view, this is a remarkable case of a far backside-propagating EUV front observed over the limb. The average EIT wave speed determined by Prise et al. (2014) using an intensity-profile technique, applied to a wave sector propagating south-west, was $221 \pm 15 \mathrm{~km} \mathrm{~s}^{-1}$, while Park et al. (2013) obtained an average speed of $473 \mathrm{~km} \mathrm{~s}^{-1}$ between the AR and the STEREO-A nominal magnetic footpoint. 

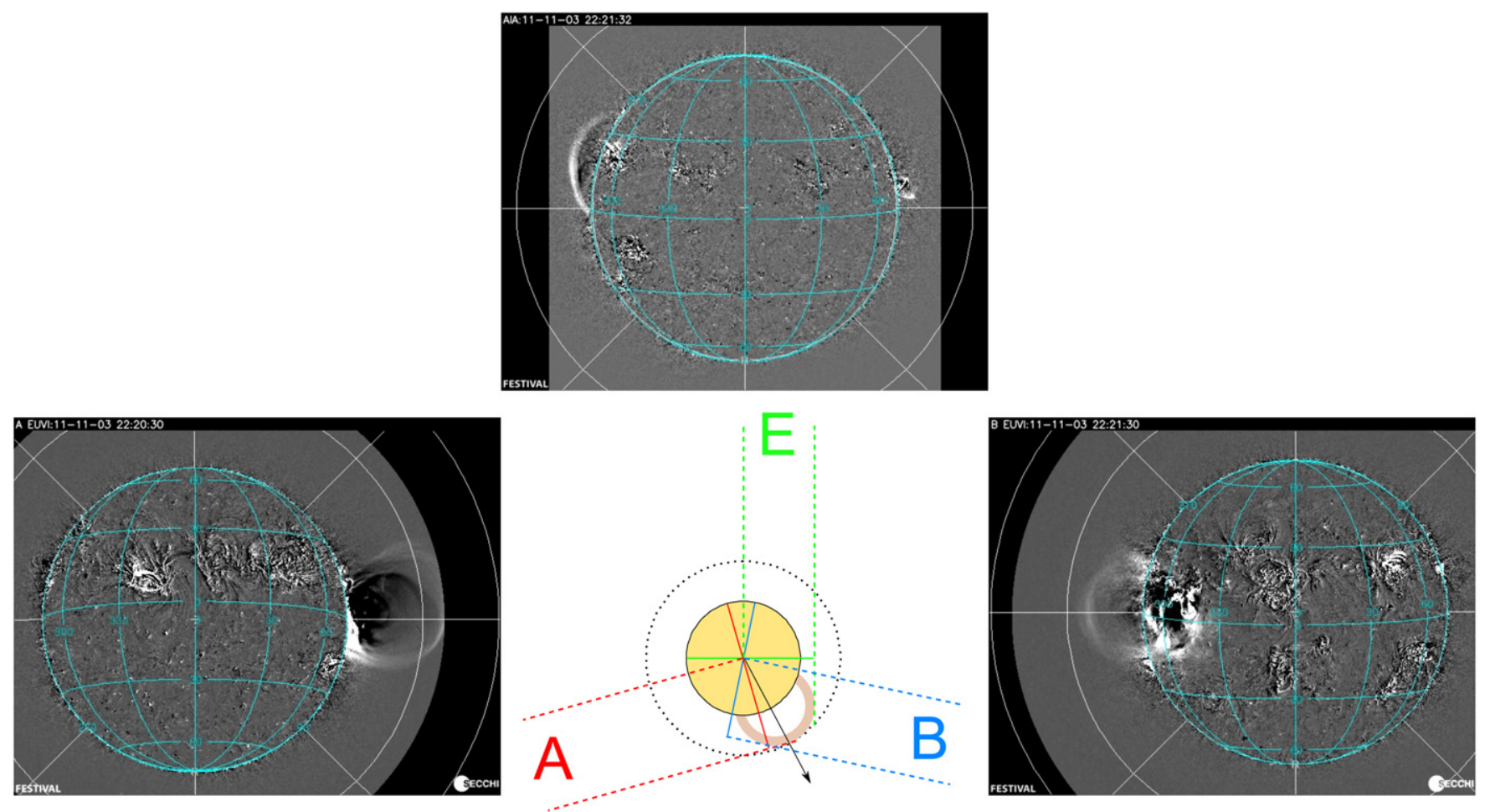

Figure 4. STEREO-A/EUVI $195 \AA ̊$, STEREO-B/EUVI $195 \AA ̊$, and SDO/AIA $193 \AA$ Å difference images at 22:20:30 UT, 22:21:31 UT, and 22:21:33 UT, respectively. The cartoon in the center outlines the approximate geometry of the EUV "dome" structure observed over the solar limbs by the three s/c. The external dotted circle corresponds to 1.7 solar radii (EUVI field of view radius).

The M4.7-X1.4 flare peaking at 22:41 UT was accompanied by a fast halo $\mathrm{CME}$, which appeared over the west solar limb at the STEREO-A/COR1 field of view at 22:22 UT (see top right panel in Figure 3) and over the east limb at STEREO-B/COR1 field of view at 22:26 UT. The angular width of the CME on STEREO-A/COR2 is $216^{\circ}$ according to the Cactus CME list (http://secchi.nrl.navy.mil/cactus/). The corresponding backside halo CME from Earth's point of view appeared in the LASCO/C3 field of view (heliocentric distance of 9.71 solar radii) at 23:30 UT. According to the LASCO CME Catalog (http://cdaw.gsfc.nasa.gov/CME_list/), the planeof-sky CME speed was $991 \mathrm{~km} \mathrm{~s}^{-1}$. Prise et al. (2014) estimated a radial speed of $972 \mathrm{~km} \mathrm{~s}^{-1}$ from STEREO-A coronograph observations and a lateral expansion speed of $674 \pm 38 \mathrm{~km} \mathrm{~s}^{-1}$, concluding that while the EIT wave was too slow to explain the SEP timing, the CME lateral expansion was consistent with the SEP release times at STEREO-A and STEREO-B footpoints (but not for Earth).

\subsection{In situ Plasma and Magnetic Field Observations}

Figure 5 shows a summary of in situ plasma and magnetic field observations from STEREO-B (left panel), $A C E$ and $S O H O$ (central panel), and STEREO-A (right panel) from 2011 November 2 (day 306 of year) to November 10 (day 314 of year). Each panel shows from top to bottom: solar wind proton speed, proton density, proton temperature, magnetic field magnitude, magnetic field vector latitudinal angle, and magnetic field vector azimuthal angle in the Radial Tangential Normal (RTN) coordinate system. The color bands in the magnetic field magnitude panel indicate the in situ magnetic field polarity estimated from the magnetic field azimuth (red denotes inward polarity, green outward polarity and intervals with field orientations close to perpendicular to the nominal
Parker spiral are marked in yellow). The arrows in the bottom $\mathrm{X}$-axis mark the SEP onset time for relativistic electrons at each s/c (see Section 4.1). When the SEP event started, STEREO-B and STEREO-A were in regions of negative magnetic field polarity (red), while $A C E$ was in a positive polarity sector (green). The three s/c were embedded in slow solar wind streams $\left(V_{\mathrm{SW}}<350 \mathrm{~km} \mathrm{~s}^{-1}\right)$.

STEREO-A observed a pair of IP shocks on November 5 21:12 UT (forward shock) and on November 6 12:35 UT (reverse shock), bounding a stream interaction region (SIR) corresponding to the density increase from late November 5 to early November 6 . These shocks are indicated by vertical dashed lines in the right panel of Figure 5. A second, closely spaced SIR observed in November 7 was not accompanied by developed shocks at $1 \mathrm{AU}$.

According to the near-Earth ICME list by Richardson and Cane, ICME transits were identified between November 2, 01:00 UT and November 3, 04:00 UT and during November 7, 17:00-23:00 UT (indicated by shaded areas in the center panel of Figure 5). November 2 ICME was already beyond $1 \mathrm{AU}$ at the time of particle onset (based on solar wind speed, its trailing edge would have been 0.17 AU beyond Earth). In situ speed during the November 7 ICME was $312 \mathrm{~km} \mathrm{~s}^{-1}$. Assuming an average propagation speed in the range $312-450 \mathrm{~km} \mathrm{~s}^{-1}$, the inferred launch window would be between early November 2 and late November 3. The parent $\mathrm{CME}$ is uncertain, but $\mathrm{CME}$ activity registered by STEREO/COR1 (see http://cor1.gsfc.nasa.gov/catalog/) points to one faint candidate CME observed over the east limb by STEREO-A and over the west limb by STEREO-B on November 3, 21:05 UT.

STEREO-B observed an interplanetary shock before the SEP event onset, on November 2, 22:08 UT (first vertical dashed line in Figure 5 left panel). Another IP shock was observed by 

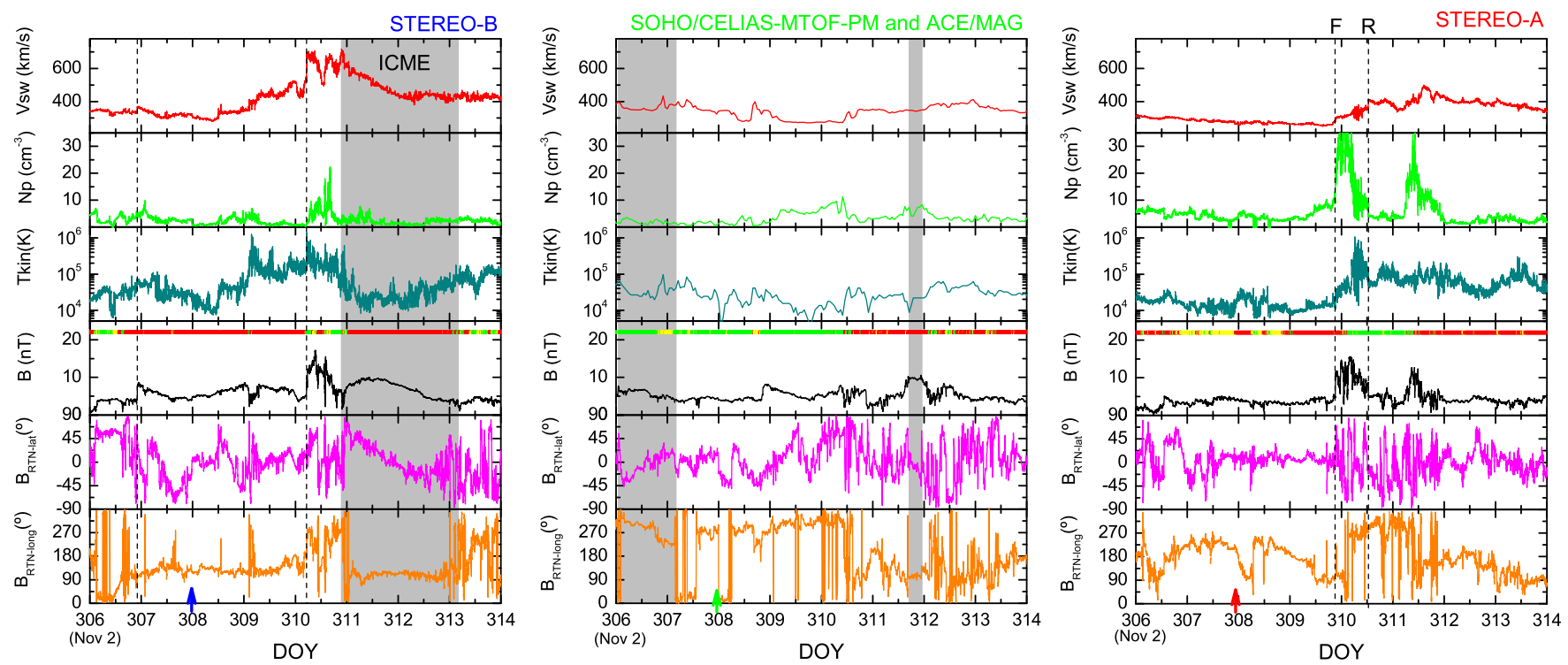

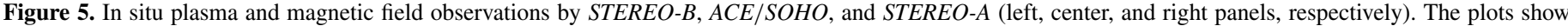

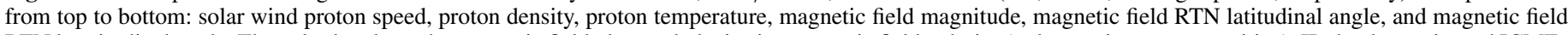

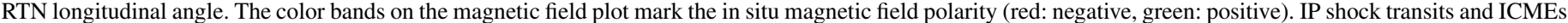
are marked by dashed lines and shaded areas, respectively. SEP onset times for relativistic electrons are marked by arrows in the bottom X-axis.

STEREO-B on November 6, 05:11 UT (second vertical dashed line). This quasi-parallel shock $\left(\theta_{B n}=29.1\right.$, http://www-ssc. igpp.ucla.edu/forms/stereo/stereo_level_3.html) was followed by an ICME from November 6, 22:50 to November 9, 04:00 UT (shaded area in the left panel of Figure 5). Although the magnetic field enhancement was moderate, this structure showed clear ICME signatures including low proton temperature, decreasing solar wind speed, smooth magnetic field rotation, and bidirectional suprathermal electron fluxes (see STEREO/SWEA pitch angle distributions at http://stereo.cesr.fr/plots.php). Assuming that this ICME is the interplanetary counterpart of the CME launched at 22:22 UT on November 3 (as seen by STEREO-A/COR1), the average shock and ICME transit speeds from the Sun to STEREO-B would be $825 \mathrm{~km} \mathrm{~s}^{-1}$ and $623 \mathrm{~km} \mathrm{~s}^{-1}$, respectively. The orientation of the flux-rope axis obtained from a minimum variance analysis of the magnetic field data during the period DOY 311.197-312.077 is along the RTN direction $(-0.28,0.91,0.31)$, meaning that its angle with respect to the radial direction $\left(X_{\mathrm{RTN}}\right)$ was $106^{\circ}$.

Figure 6 shows magnetic field observations by MESSEN$G E R$, in orbit around Mercury. The light blue shaded areas delimit intervals when MESSENGER observed structures related to Mercury's magnetosphere identified by B. Anderson (JHU/APL, 2013, private communication). Outside these intervals, the s/c was in the solar wind. MESSENGER observed an IP shock transit on November 4 15:09 UT (vertical dashed line in the figure), followed by a region showing ICME signatures such as enhanced field and magnetic field rotation, from November 5 00:30 to 18:00 UT (gray shaded area in the figure). Taking into account that the heliocentric distance of MESSEN$G E R$ was $0.44 \mathrm{AU}$, if the IP shock and the ICME originated during the launch of the CME at 22:22 UT on November 3 (the same ICME observed in situ by STEREO-B on November 6-9), the average transit speed to travel from the Sun to MESSENGER would be $1090 \mathrm{~km} \mathrm{~s}^{-1}$ for the shock, and $\sim 700 \mathrm{~km} \mathrm{~s}^{-1}$ for the ICME. The orientation of the flux rope axis obtained from a minimum variance analysis during the period DOY 309.135309.571 (excluding the magnetospheric crossing during $309.23-309.50)$ is along the RTN direction $(-0.15,0.96,0.25)$,

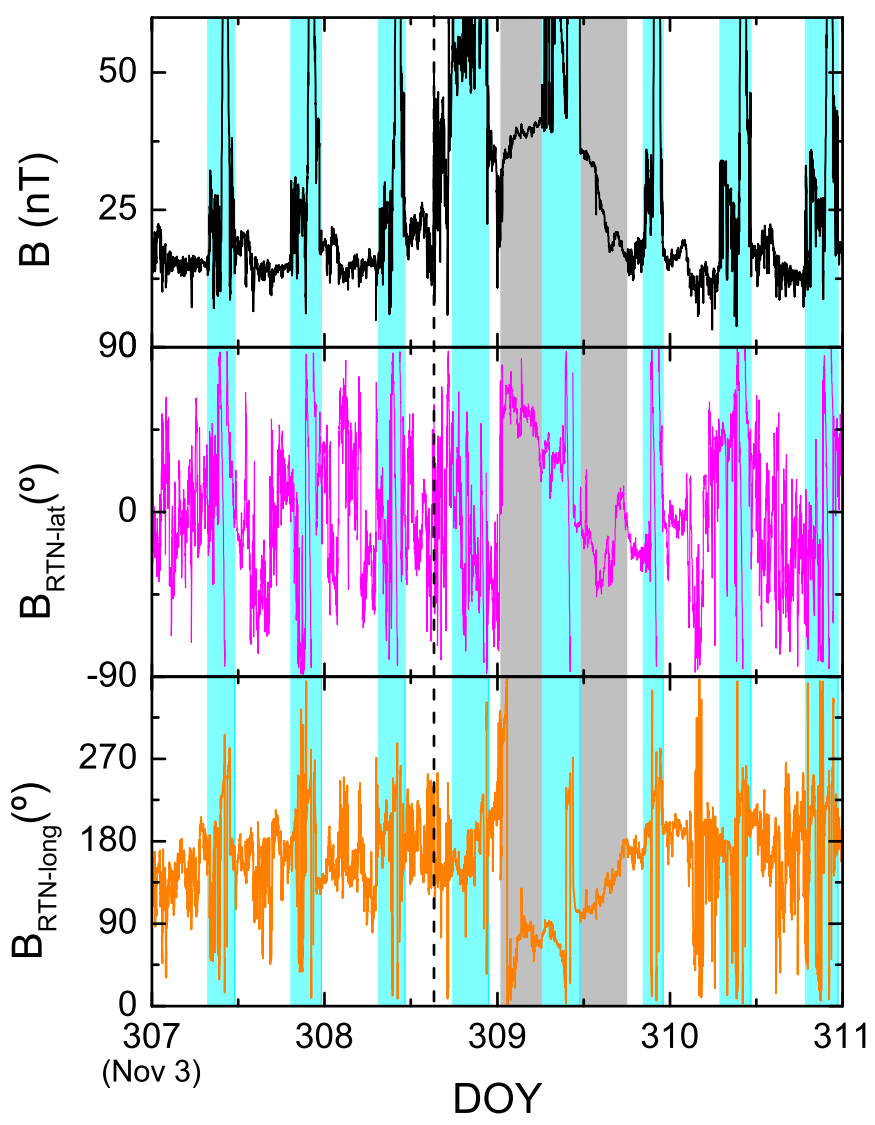

Figure 6. In situ magnetic field observations by MESSENGER. From top to bottom: magnetic field magnitude, magnetic field RTN latitudinal angle, and magnetic field RTN longitudinal angle. IP shock and ICME are marked by a black dashed line and a gray shaded area, respectively. Light blue shaded areas mark time intervals when the s/c was inside Mercury's magnetosphere.

$99^{\circ}$ from the radial direction. Given the small longitudinal and latitudinal separation between $S T E R E O-B$ and MESSENGER at the time of the ICME transit $\left(6^{\circ}\right.$ and $3^{\circ}$, respectively) and assuming a radial propagation for the ICME, we infer that both 


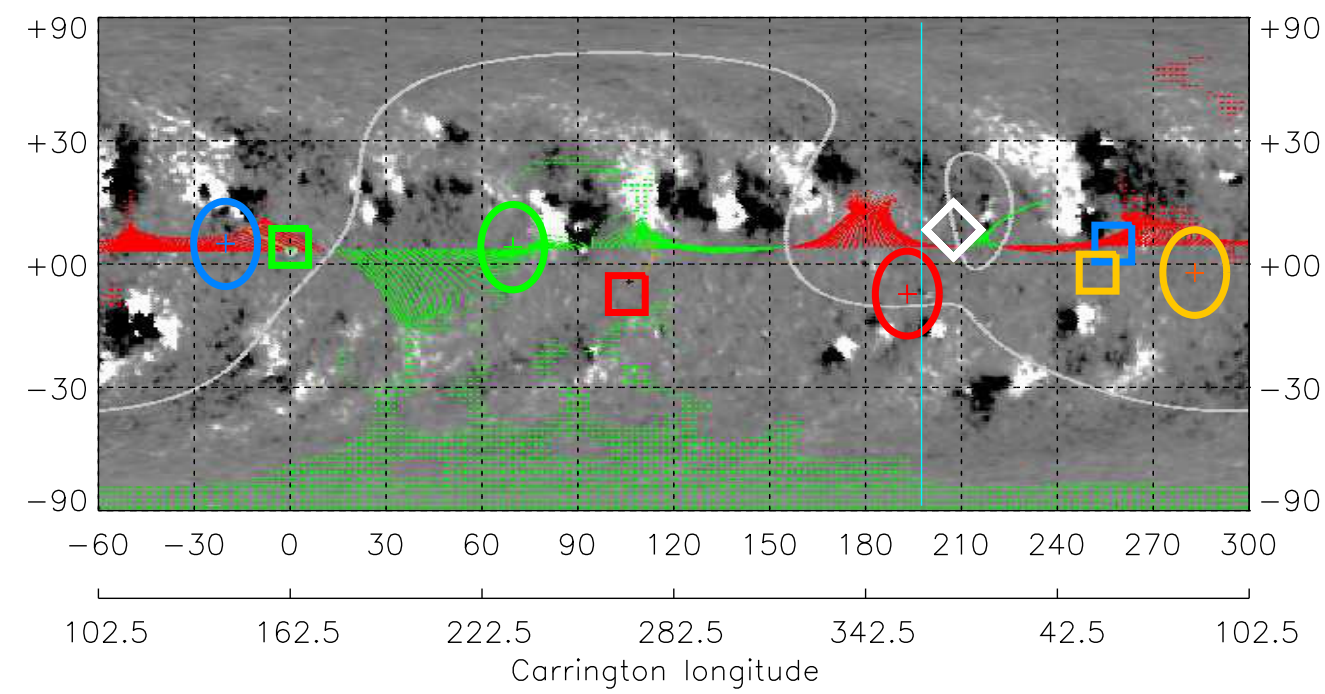

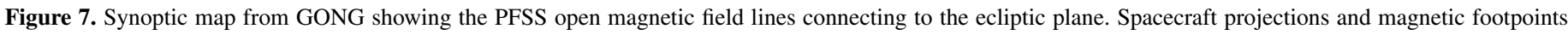

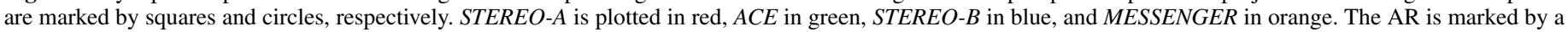
white diamond.

$\mathrm{s} / \mathrm{c}$ crossed essentially the same section of the ICME, showing a significant expansion with radial distance and comparable flux rope orientation.

\subsection{Magnetic Connectivity}

Figure 7 shows a synoptic map from GONG with open field lines connecting to the ecliptic plane computed using the PFSS model, overplotted on top of a solar magnetogram taken at 22:14 UT on November 3 (http://gong.nso.edu/ data/magmap/pfss.html). The source surface is fixed at a height of $2.5 R_{\odot}$. Red denotes negative polarity and green positive polarity. The white diamond marks the location of the flaring AR. The polarity separator (heliospheric current sheet) is marked by the white wavy line and the dotted areas correspond to coronal holes. STEREO-A, STEREO-B, ACE, and MESSENGER projections (radial mapping of the s/c to the source surface) are marked by red, blue, green, and orange squares, respectively. Their corresponding nominal magnetic footpoints along the Parker spiral are marked by circles. These footpoints have been calculated using the one-hour averaged solar wind speed measured in situ at the time of electron onset: 321,346 , and $267 \mathrm{~km} \mathrm{~s}^{-1}$ for STEREO-B, ACE, and STEREO-A, respectively. A value of $350 \mathrm{~km} \mathrm{~s}^{-1}$ has been assumed for MESSENGER, with no in situ solar wind measurements available. The corresponding ideal Parker spiral lengths are 1.37, 1.18, 1.25, and $0.46 \mathrm{AU}$ for STEREO-B, ACE, STEREO-A, and MESSENGER, respectively. Although the PFSS model uses several days old magnetograph data for the backside hemisphere, the polarities match well the in situ observations described in Figure 5, supporting that PFSS results offer an adequate overall description of the large-scale coronal magnetic fields. For a detailed discussion on the limitations and accuracy of PFSS models to extrapolate the photospheric magnetic field to the corona, we refer the reader to Nitta \& De Rosa (2008) and references therein. Table 1 shows the location of the spacecraft projections, nominal footpoints and their separation with respect to the flare location.

\subsection{In situ Energetic Particle Observations}

Figure 8 shows an overview of energetic particle observations from STEREO-B, Earth (ACE and SOHO), and STEREO-A,
Table 1

Spacecraft Locations, Footpoints, and Separation with Respect to the AR

\begin{tabular}{lcccccc}
\hline \hline & $\begin{array}{c}R^{\mathrm{a}} \\
(\mathrm{AU})\end{array}$ & $\phi^{\mathrm{a}}$ & $\theta^{\mathrm{a}}$ & $\phi_{f}{ }^{\mathrm{a}}$ & $\Delta \phi^{\mathrm{a}}$ & $\Delta \alpha^{\mathrm{a}}$ \\
\hline STEREO-A & 0.97 & 267.8 & -7.0 & 356 & 14 & 21 \\
\hline ACE & 0.98 & 162.5 & 4.0 & 232 & 138 & 136 \\
\hline STEREO-B & 1.09 & 60.0 & 4.8 & 142 & 132 & 130 \\
\hline MESSENGER & 0.44 & 55.1 & -2.0 & 85 & 75 & 75 \\
\hline $\mathrm{AR}^{\mathrm{b}}$ & $\cdots$ & 10 & 8 & $\ldots$ & $\ldots$ & $\ldots$
\end{tabular}

Notes.

a $R$ : heliocentric distance in $\mathrm{AU} ; \phi$ : Carrington longitude; $\theta$ : heliographic latitude; $\phi_{f}$ : nominal magnetic footpoint's Carrington longitude; $\Delta \phi$ : longitudinal separation footpoint to AR; $\Delta \alpha$ : angular separation footpoint to AR. All the angular values are in degrees.

b AR location has been marked at the ribbon brightening, rather than at the top of the erupting loop.

during 2011 November 2-10. Omnidirectional one-hour averaged electron and proton fluxes observed in different energy bands are shown in the top two plots, while the bottom plots show the temporal evolution of the ${ }^{4} \mathrm{He}$ to proton and $\mathrm{Fe} / \mathrm{O}$ abundance ratios. Electron fluxes below $500 \mathrm{keV}$ (from STEREO/ SEPT and ACE/EPAM-LEFS60) are prone to ion contamination, which becomes significant during periods of strongly enhanced flux of sub-MeV protons, for instance around the shock passage at STEREO-B on November 6 or during the CIR-associated shock-pair passage at STEREO-A. The intervals with contaminated electron fluxes have been plotted using dashed lines. The three spacecraft observed velocity dispersion during the proton event onset. In all cases, energetic electrons and $>30 \mathrm{MeV}$ protons reached the maximum intensity within few hours after the onset, with a faster rise at STEREO-A and then showed an exponential decay (see detailed onset timing information in Section 4.1). STEREO-A registered the highest intensities for all energies and species, with the exception of the local enhancement after the shock passage at STEREO-B, which yielded the highest fluxes for protons below $4 \mathrm{MeV}$. Low-energy $(<10 \mathrm{MeV})$ proton intensity at STEREO-B matches the expected 

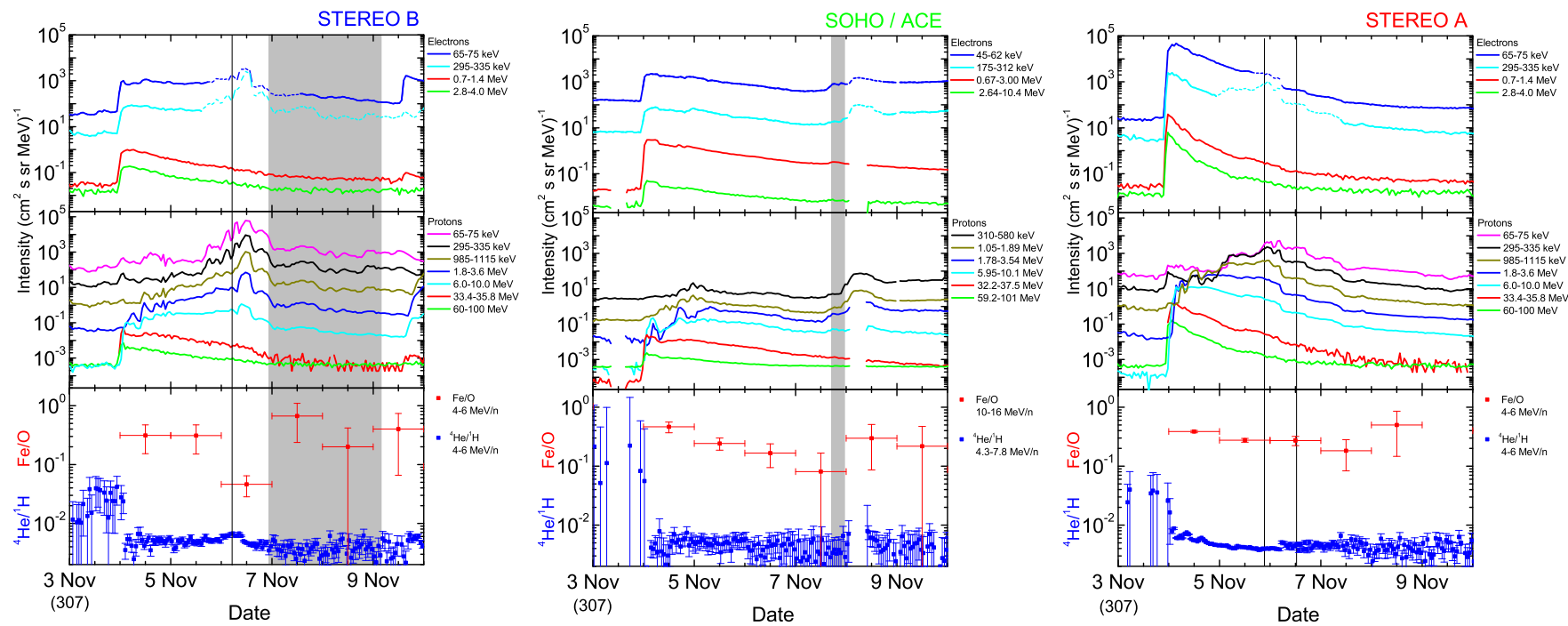

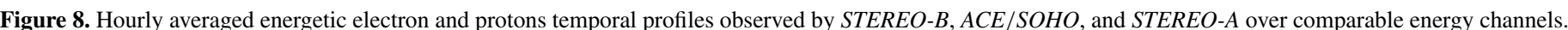

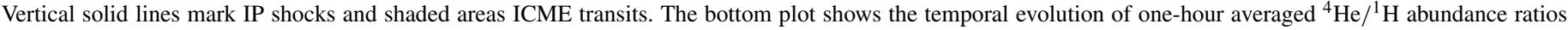

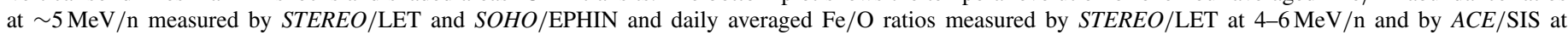
$10-15 \mathrm{MeV} / \mathrm{n}$. Vertical error bars represent statistical uncertainties.

behavior for a gradual event observed from a location westward from the source region, progressively better connected to the central region of the IP shock as it approaches 1 AU and reaching the best connection after the shock passage (see, e.g., Cane et al. 1988). The local shock had little influence for relativistic electrons and for protons above $10 \mathrm{MeV}$, which showed very similar temporal profiles at STEREO-B and Earth, with a factor of $\sim 2$ higher intensities at STEREO-B compared to Earth (see quantitative comparisons in Section 4.4).

The ${ }^{4} \mathrm{He} /{ }^{1} \mathrm{H}$ abundance ratios at $\sim 5 \mathrm{MeV} / \mathrm{n}$ (blue solid squares at the bottom plots in Figure 8) show nearly constant values close to 0.005 during the course of the event for Earth and STEREO-B, with an enhancement up to 0.006-0.007 during the shock passage at $S T E R E O-B$, followed by a depletion in the downstream region and inside the ICME. At STEREO-A the ratio was maximum during the early phase of the event, with values still small, around 0.008 early on November 4 , decreasing to an almost constant value of 0.004 during November 5 . These helium-poor values are very far of the typical $\mathrm{He} / \mathrm{H} \sim 0.1$ for impulsive SEP events and below the $\sim 0.01$ average ratios of gradual SEP events (e.g., Kallenrode 2003). $\mathrm{He} / \mathrm{H}$ ratios during gradual SEP events are influenced by different factors such as the seed population (see, e.g., Kocharov et al. 2010) and differential scattering of ${ }^{4} \mathrm{He}$ and ${ }^{1} \mathrm{H}$ by protongenerated waves (Reames \& Ng 2002). Solar flares are very unlikely to contribute material with suppressed $\mathrm{He} / \mathrm{H}$ (Reames $\& \mathrm{Ng} 2002$ ).

Red solid squares at the bottom plots in Figure 8 show $\mathrm{Fe} / \mathrm{O}$ ratios measured by STEREO at $4-6 \mathrm{MeV} / \mathrm{n}$ and by $A C E / \mathrm{SIS}$ at $10-15 \mathrm{MeV} / \mathrm{n}$. Daily averages have been used due to the statistical limitations of the data. STEREO-A observed values decaying from 0.38 on November 4 to 0.27 on November 6. STEREO-B registered constant values $\sim 0.3$ on November 4 and 5 and a statistically significant drop down to 0.05 during November 6 . ACE observed Fe/O $\sim 0.46$ during November 4, decaying to 0.17 on November 6. These $\mathrm{Fe} / \mathrm{O}$ values lie within the wide range of variation typically observed during gradual events (see Desai et al. 2006) and below the average values for impulsive ${ }^{3} \mathrm{He}$-rich events (see Mason et al. 2004).

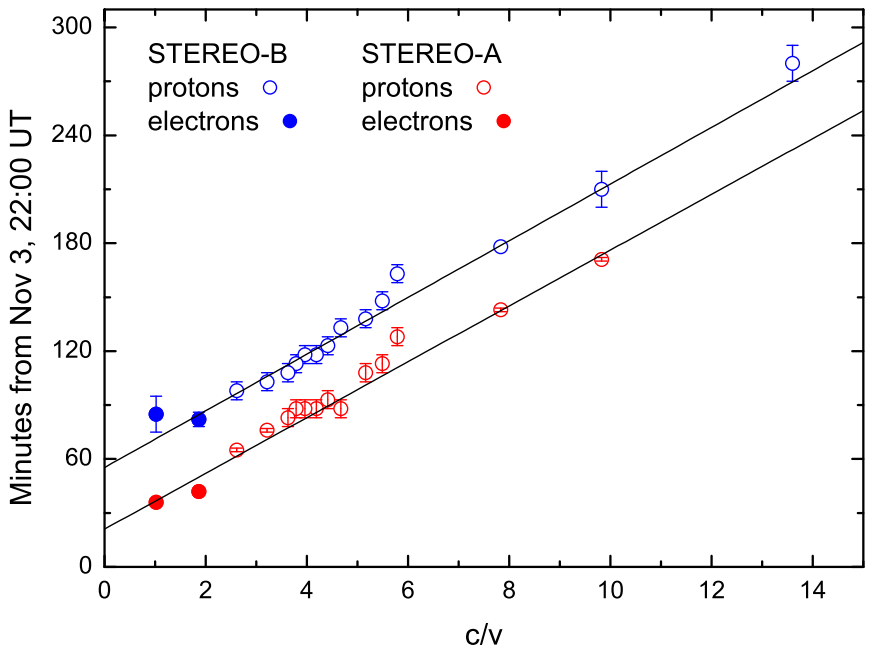

Figure 9. Velocity dispersion analysis and linear fits for STEREO-A and STEREO-B.

\section{DATA ANALYSIS}

\subsection{Energetic Particle Timing}

Prise et al. (2014) presented a multi-point Velocity Dispersion Analysis (VDA) of the SEP event based on onset times determined using the traditional n-sigma threshold approach (see Krucker et al. 1999). We have determined onset times for electron and proton data from the SEPT, HET, and LET instruments on board STEREO $A$ and $B$, using the Poisson-CUSUM approach described by Huttunen-Heikinmaa et al. (2005), which generally provides more robust onset detection capabilities.

The resulting VDA plots and linear fits are shown in Figure 9 and, as an example, Figure 10 shows the rising phases of one of the nearly relativistic electron channels of the instruments STEREO-A/SEPT, STEREO-B/SEPT, and ACE/EPAMLEFS60. The fit parameters (effective path length, $L$ and inferred release time at the Sun (SRT)) are listed in the second and third columns of Table 2 (for a discussion on the reliability of the VDA technique, see Sáiz et al. 2005; Lintunen \& 


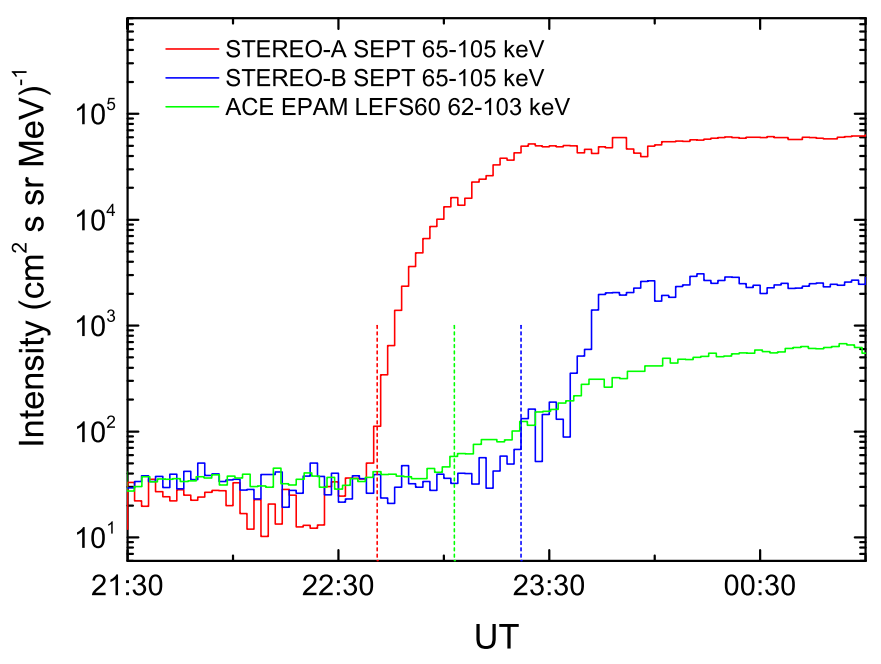

Figure 10. Rising phase of nearly relativistic electrons observed by STEREO-A, $S T E R E O-B$, and ACE. Onset times are marked by vertical dashed lines.

Table 2

Velocity Dispersion Analysis Results

\begin{tabular}{lcccc}
\hline \hline & $\begin{array}{c}L^{\mathrm{a}} \\
(\mathrm{AU})\end{array}$ & $\begin{array}{c}\mathrm{SRT}^{\mathrm{b}} \\
(\mathrm{UT})\end{array}$ & $\begin{array}{c}t_{0} \mathrm{c} \\
(\mathrm{UT})\end{array}$ & $\begin{array}{c}h^{\mathrm{d}} \\
\left(R_{\odot}\right)\end{array}$ \\
\hline STEREO $-A$ & $1.87 \pm 0.05$ & $22: 21 \pm 2 \mathrm{~min}$ & $22: 29$ & 2.3 \\
\hline STEREO $-B$ & $1.90 \pm 0.06$ & $22: 55 \pm 4 \mathrm{~min}$ & $23: 04$ & 5.2 \\
\hline Earth & $1.86 \pm 1.44^{\mathrm{e}}$ & $22: 47 \pm 15 \mathrm{~min}^{\mathrm{e}}$ & $22: 55$ & 4.4 \\
\hline
\end{tabular}

Notes.

a Effective propagation path length.

b Solar release time (at the Sun).

${ }^{c}$ Solar release time shifted by light propagation time to $1 \mathrm{AU}$ in order to compare with electromagnetic observations.

d CME height at the time of particle release.

e Results for Wind electrons obtained by Prise et al. (2014).

Vainio 2004; Kahler \& Ragot 2006; Reames 2009a; Rouillard et al. 2012; Vainio et al. 2013). The results agree within error bars with the values found by Prise et al. (2014). During this period, the $\mathrm{SOHO}$ s/c was rotated $180^{\circ}$ with respect to its nominal pointing, meaning that the fields of view of all particle instruments were looking perpendicular to the nominal Parker spiral direction. Since first arriving particles tend to propagate field-aligned (see Section 4.2), SOHO data would yield delayed onset times. For this reason and since $\mathrm{SOHO}$ is required to cover the high-energy proton and electron observations at $1 \mathrm{AU}$, we did not attempt a VDA for near-Earth s/c. A reduced VDA fit, using only Wind electron data, performed by Prise et al. (2014) yielded a path length of $1.86 \pm 1.44$ and an SRT of 22:47 \pm 15 minutes MESSENGER/EPPS has a similar problem due to its anti-sunward pointing direction (Anderson et al. 2007) that does not allow us to estimate the time of the first arriving particles at $0.44 \mathrm{AU}$. The 141-224 keV electron flux onset was observed by MESSENGER at 23:07 UT \pm 5 minutes. Since this value corresponds to sunward-propagating electrons, it is not valid for SRT estimation; however, it sets an upper limit for the SRT of 23:01 UT (23:09 UT when shifted by the light-time travel to $1 \mathrm{AU})$.

The fourth column in Table 2 lists the SRT values shifted by the light travel time to $1 \mathrm{AU}$, in order to compare with the electromagnetic observations. The last column shows the $\mathrm{CME}$ heliocentric height at the time of particle release, obtained from STEREO-A/COR1 observations of the CME over the west solar limb. According to the VDA analysis results, and interpreting the delays in terms of delayed injection close to the Sun, energetic particles were released first at magnetic field lines connecting to STEREO-A, when the leading edge of the CME was at 2.3 solar radii, then to Earth, when the leading edge of the CME was at 4.4 solar radii and shortly after at magnetic field lines connecting to STEREO-B (and MESSENGER) when the leading edge of the CME was already at 5.2 solar radii. Note that according to this analysis, it takes less than 40 minutes to get the particles injected over a broad longitudinal range connecting to the four locations $\left(270^{\circ}\right.$, see footpoint separation in Figure 1). Reames (2009b) studied the CME-driven shock heights at the time of energetic particle release during ground level enhancements (GLEs) and found that for well-connected events the particle release begins when the shock reaches radial distances of $\sim 2$ solar radii. Analogously, according to Gopalswamy et al. (2012), the release of GLE particles for well-connected events occurs when the CMEs reach an average height of $\sim 3$ solar radii. For poorly connected events, the average values of the CME heights at the time of particle release is larger, which has been interpreted as an indication that the $\mathrm{CME}$ has to expand further before the shock crosses the field line connecting to the observer (Reames 2009a, 2009b; Gopalswamy et al. 2012).

According to the analysis by Prise et al. (2014), the lateral expansion speed of the CME $\left(674 \pm 38 \mathrm{~km} \mathrm{~s}^{-1}\right)$ was not fast enough to explain the early release of energetic particles at Earth's footpoint. In order to estimate the speed required for an azimuthally propagating source to explain the particle timing, Richardson et al. (2014) divided the angular distance between STEREO-A and SOHO footpoints by the onset delay of 24-41 MeV protons at Earth compared to STEREO-A, inferring an estimated angular speed of 5.3 minute ${ }^{-1}$, which corresponds to a speed of $1070 \mathrm{~km} \mathrm{~s}^{-1}$ over the solar surface. A similar calculation using the release times presented in Table 2 provides an estimated angular speed of 4.8 minute $^{-1}$ (equivalent to a minimum speed of $964 \mathrm{~km} \mathrm{~s}^{-1}$, when a height of 1 solar radii is assumed).

\subsection{Energetic Particle Anisotropies}

The SEPT instruments on board the three-axis stabilized s/c STEREO consist of four apertures pointing sunward along the Parker spiral, anti-sunward along the Parker spiral, North and South. The directional information provided by these four sectors can be used to reconstruct the electron pitch-angle distribution. The pitch-angle coverage is limited compared with rotating s/c such as Wind, and varies depending on the orientation of the local magnetic field vector. Figure 11 shows anisotropy information for nearly relativistic electrons observed by STEREO-A (left panel), Wind (center panel), and STEREO-B (right panel). The plots show from top to bottom: (1) pitchangle distribution with color-scaled intensity and divided in four pitch-angle bins, (2) orientation of each instrument sector in the pitch-angle space, (3) intensity registered by each sector, and (4) first-order anisotropy index (intervals in gray correspond to periods at background level, when the anisotropy index has no meaning. Intervals with pitch-angle coverage under $45^{\circ}$ have been excluded, introducing artificial data gaps). STEREO plots correspond to 55-105 keV electrons and Wind plot to 50-82 keV electrons. The first-order anisotropy index $A$ is defined by

$$
A=\frac{\int_{-1}^{+1} \mu f(\mu) d \mu}{\int_{-1}^{+1} f(\mu) d \mu},
$$



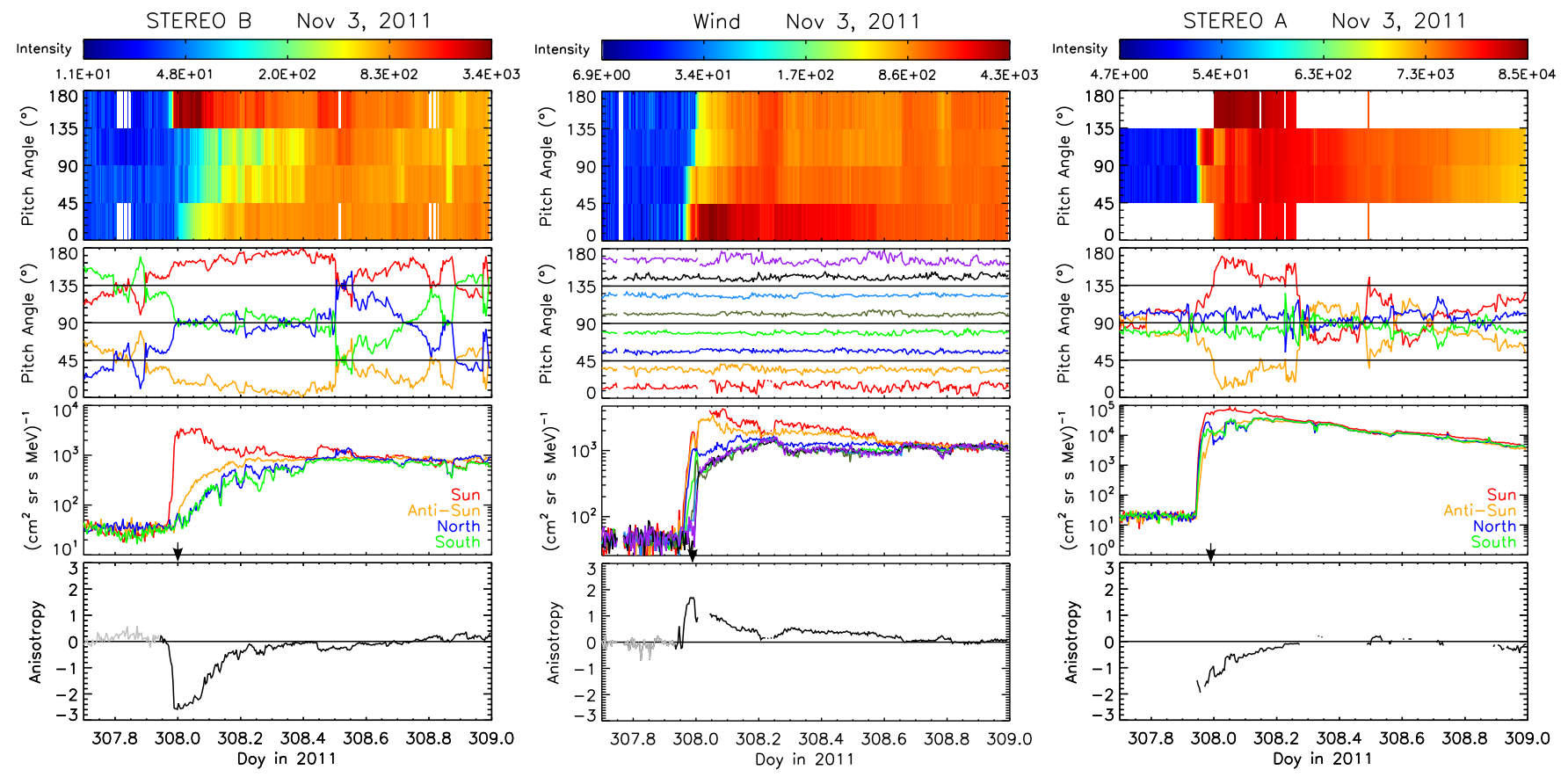

Figure 11. Temporal evolution of electron pitch-angle distributions measured by STEREO-A/SEPT, Wind/3DP, and STEREO-B/SEPT (see text for details).
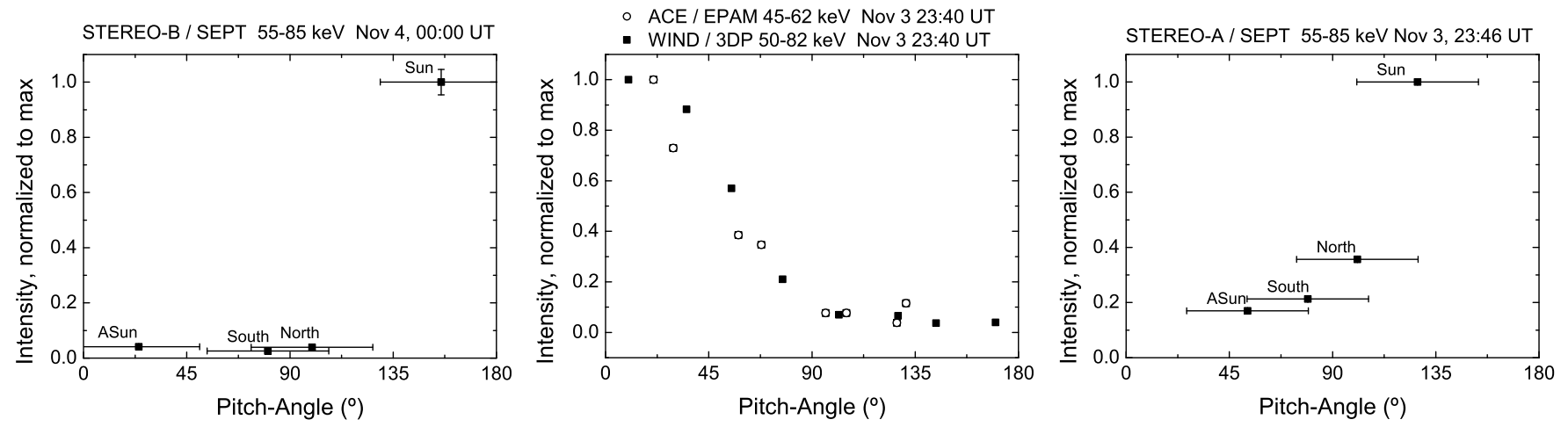

Figure 12. Snapshots of electron pitch-angle distributions from STEREO-B (left panel), ACE, and Wind (center panel) and STEREO-A (right panel). The corresponding times are marked by arrows under the third panel in Figure 11.

where $\mu$ is the pitch-angle cosine and $f(\mu)$ the pitch-angle distribution, which is estimated using a second-order polynomial fit for each five-minute averaged pitch-angle distribution (using four points in the case of STEREO/SEPT and eight points for Wind/3DP). Figure 12 shows snapshots of the pitch-angle distribution at the times marked by arrows under the third plots in Figure 11.

STEREO-A 's pitch-angle coverage is seriously limited during the event onset, improving as the event progresses, while STEREO-B and Wind offer good coverage during the whole event. In spite of this limitation, data show clearly that the three $\mathrm{s} / \mathrm{c}$ observed anisotropic electron fluxes lasting for several hours during the rising phase of the event and in the early decay phase. The examination of proton pitch-angle distributions (not shown in the figure) also reveals clear anisotropies during the early phase of the event for STEREO-A, STEREO-B, and Earth.

Electron anisotropies at $S T E R E O-B$ were particularly large and showed a clear indication of reflected particles. This backscattered SEP population is identified in the top plot of Figure 11 by the flux increase at the pitch-angle sector closer to 0 (orange line in the second plot), which, given the negative polarity, corresponds to particles propagating sunward. This is also seen in the third panel showing the sector intensities (orange line representing the intensity measured by the anti-Sun telescope). In contrast to the progressive isotropization observed by STEREO-A and Wind, the anti-sunward pointing telescope of STEREO-B observed an increase well before the telescopes pointing North and South, that stayed enhanced during several hours. The first IP shock marked in the right panel of Figure 5, observed in situ by STEREO-B on November 2, 22:08 UT, could provide the structure acting as a reflecting barrier located beyond STEREO-B at the time of the SEP event onset. This structure may create the appropriate conditions to mirror energetic particles back to the observer in sunward direction, as previously suggested by different authors (Anderson et al. 1995; Bieber et al. 2002; Malandraki et al. 2002; Roelof 2008; Tan et al. 2009; Agueda et al. 2010; Klassen et al. 2012). Note that this reflecting boundary may have also contributed to the observation of sunward-directed particles by MESSENGER.

Strong anti-sunward anisotropies are commonly interpreted as an evidence of both a direct injection of energetic particles at the observers' magnetic footpoint and weak scattering during their IP transport. On the contrary, the combination of long onset delays, low anisotropies, and poor nominal magnetic connection 
is often interpreted as evidence of cross-field IP diffusion (e.g., Dresing et al. 2012). This does not seem to be the case for the 2011 November 3 SEP event.

\subsection{Interplanetary Transport Simulations}

In order to have further insights on the temporal evolution of the electron release close to the Sun, we make use of a Monte Carlo interplanetary propagation model developed by Agueda et al. (2008) combined with an inversion procedure to fit the in situ SEP observations (Agueda et al. 2014). The Monte Carlo transport model is based on the focused transport equation, including the effects of scattering by magnetic fluctuations, focusing, convection, and adiabatic deceleration. Only parallel transport along magnetic field lines is considered. The model assumes an Archimedean spiral magnetic flux tube connecting the Sun and the spacecraft. The value of the solar wind speed measured by the spacecraft is used to estimate the curvature of the Parker spiral and constrain the global scenario. A turbulent component is assumed to be superposed onto the large structure of the IMF. In this work, we use the radial mean free path, $\lambda_{r}$, to describe the pitch-angle scattering processes undergone by the energetic particles. Based on previous works (Palmer 1982; Kallenrode et al. 1992; Ruffolo et al. 1998), we assume that it is a good approximation to take $\lambda_{r}$ to be independent of distance. The particle source is located at an heliocentric distance of 2 solar radii and the release time history is considered to be a succession of impulsive (delta) isotropic injections. The timing and intensity of each injection episode is inferred by using an inversion approach to fit the measured directional electron intensities with the model results. Each s/c observations are independently fitted. The free parameters of the model are the radial mean free path, $\lambda_{r}$, and the injection profile at the Sun (details can be found in Agueda et al. 2014).

Figure 13 summarizes the transport model results for STEREO-B (left), ACE (center), and STEREO-A (right). The top panels show the observed sector intensities (blue solid circles) corresponding to $62-102 \mathrm{keV}$ electrons for STEREO/ SEPT and $65-105 \mathrm{keV}$ electrons for ACE/EPAM. STEREO$A$ and STEREO-B sectors 1-4 correspond to Sunward, AntiSunward, Northward and Southward pointing fields of view. A selection of six (out of eight) sectors of the LEFS60 sensor is shown for $A C E / E P A M$. The simulated sector intensities are represented by a solid black line. The small plots under each temporal profile show the pitch-angle corresponding to each sector. The three bottom panels show the injection profile at the Sun derived from the best fits. The profiles have been shifted by +500 s to allow comparison with the electromagnetic emissions. The dashed areas in the injection panels mark the time intervals when the results of the inversion should be taken with care, as release episodes occurring during these periods produce a response at the spacecraft location peaking after the selected fitting period. The best fits correspond to parallel mean free paths of 1.2, 0.7, and 0.4 AU for STEREO-B, ACE, and STEREO-A, respectively.

Under the assumptions of the model, the best fit for STEREO-A is achieved for $\lambda_{r}=0.4 \mathrm{AU}$ and an extended injection close to the Sun starting around 22:30 UT and lasting for several hours. The fit reproduces reasonably well the sectored intensities observed by SEPT, with the exception of the late increase measured by the anti-sunward pointing telescope and a transient decrease observed by the telescope pointing northward, which is likely related to local variations in the solar wind plasma.
The model reproduces very well the sector intensities observed by $A C E$, with an optimal fit for $\lambda_{r}=0.7 \mathrm{AU}$, requiring an extended injection close to the Sun, starting around 22:55 UT and showing intensities about two orders of magnitude lower than the intensities at STEREO-A. The intensity discontinuity around 00:00 UT on November 4 is caused by a sudden change in the magnetic field direction which permits a direct observation of field-aligned directions (pitch angles near $0^{\circ}$ in sector 4 ), which show the highest intensity.

Under the assumptions of the model, the best fit for STEREO-B observations is obtained for $\lambda_{r}=1.2 \mathrm{AU}$, requiring also an extended injection close to the Sun starting around 23:10 UT and with intensity levels much lower than STEREO-A. The model fails to reproduce the high intensities measured by the antisunward-pointing telescope, supporting the idea of the presence of a reflecting barrier located beyond the s/c, which has not been included in the simulation (see Agueda et al. 2010).

\subsection{Longitudinal Dependence of the Electron Peak Flux}

Following the approach by Lario et al. (2006), the broadness of the longitudinal distribution of the particles can be characterized plotting the peak intensities as a function of the longitudinal separation between each s/c footpoint and the flaring region. Since this involves data from different instruments, it requires an adequate inter-calibration of the data. Lario et al. (2013) presented inter-calibration factors for $A C E / E P A M-D E$ electrons and the E300 SOHO/EPHIN electron channel, which permit a quantitative comparison with measurements by STEREO/ SEPT and STEREO/HET. We have used these factors and preevent background subtracted intensities to obtain Gaussian fits for the longitudinal distribution of electron peak intensities at different energies:

$$
I=I_{0} \cdot \exp \left[-(\Delta \phi /(\sqrt{2} \sigma))^{2}\right]
$$

where $I$ is the peak intensity, $\Delta \phi$ the longitudinal separation between the observer's footpoint and the AR. Since we will consider only the s/c close to $1 \mathrm{AU}$, we have neglected any radial dependence in the intensity. We have also assumed that the Gaussian is centered at the optimal nominal connection $\Delta \phi=0$, thus the free fit parameters are $I_{0}$ and $\sigma$. The resulting sigmas are $45^{\circ} .3 \pm 0.7,46^{\circ} .2 \pm 0.7,46.9 \pm 0.6$, and $46^{\circ} .2 \pm 0.9$ for mean electron energies of $75 \mathrm{keV}, 133 \mathrm{keV}, 235 \mathrm{keV}$, and $1.5 \mathrm{MeV}$, respectively. As an example, the Gaussian fit for $235 \mathrm{keV}$ electron observations is shown in Figure 14. The almost energy-independent value of $\sigma \sim 46^{\circ}$ is a consequence of the similar electron spectral shapes measured at STEREO-A, $S T E R E O-B$, and near Earth. The extrapolation of the Gaussian to the borders of the longitudinal interval $\left(-180^{\circ},+180^{\circ}\right)$ for $133 \mathrm{keV}$ electrons is above typical instrumental background, meaning that the event would have been detectable over the whole longitudinal interval around the Sun. Similar analysis for protons yields $\sigma$ values of $44.6 \pm 0.3$ and $46.8 \pm 1.7$ for 14-24 MeV and 24-40.5 MeV, respectively.

\section{SUMMARY AND DISCUSSION}

On 2011 November 3, energetic particle flux enhancements were observed by spacecraft near Earth (SOHO, ACE, Wind), STEREO-A, and STEREO-B, and by MESSENGER in-orbit around Mercury. These observing locations cover a wide interval of heliolongitudes around the Sun. The examination of multipoint remote-sensing and in situ observations show strong 
STEREO-B
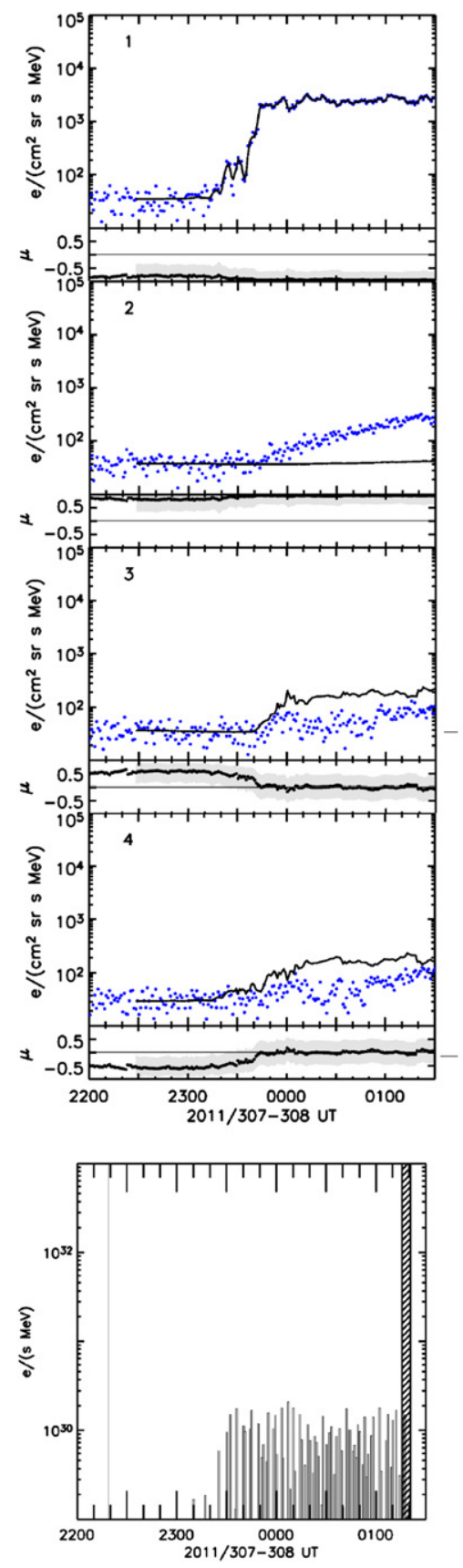

ACE
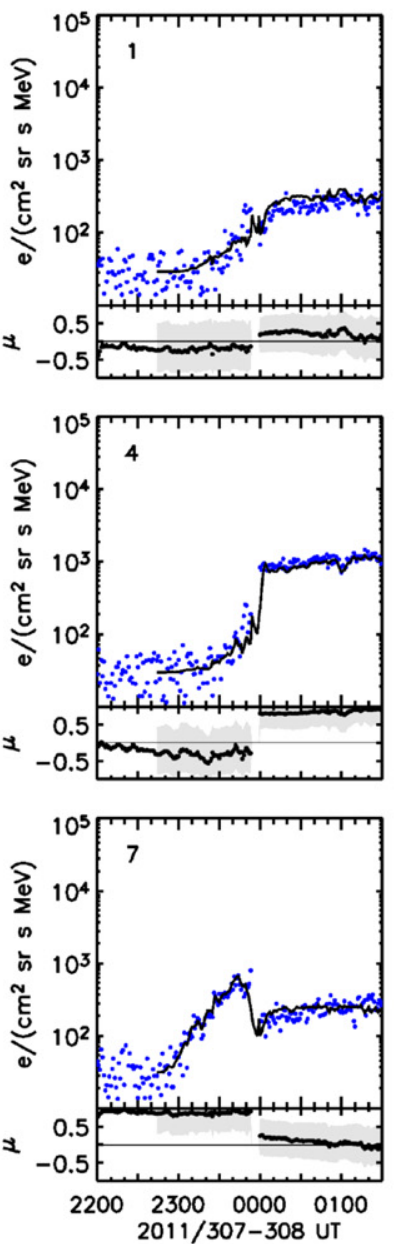

STEREO-A
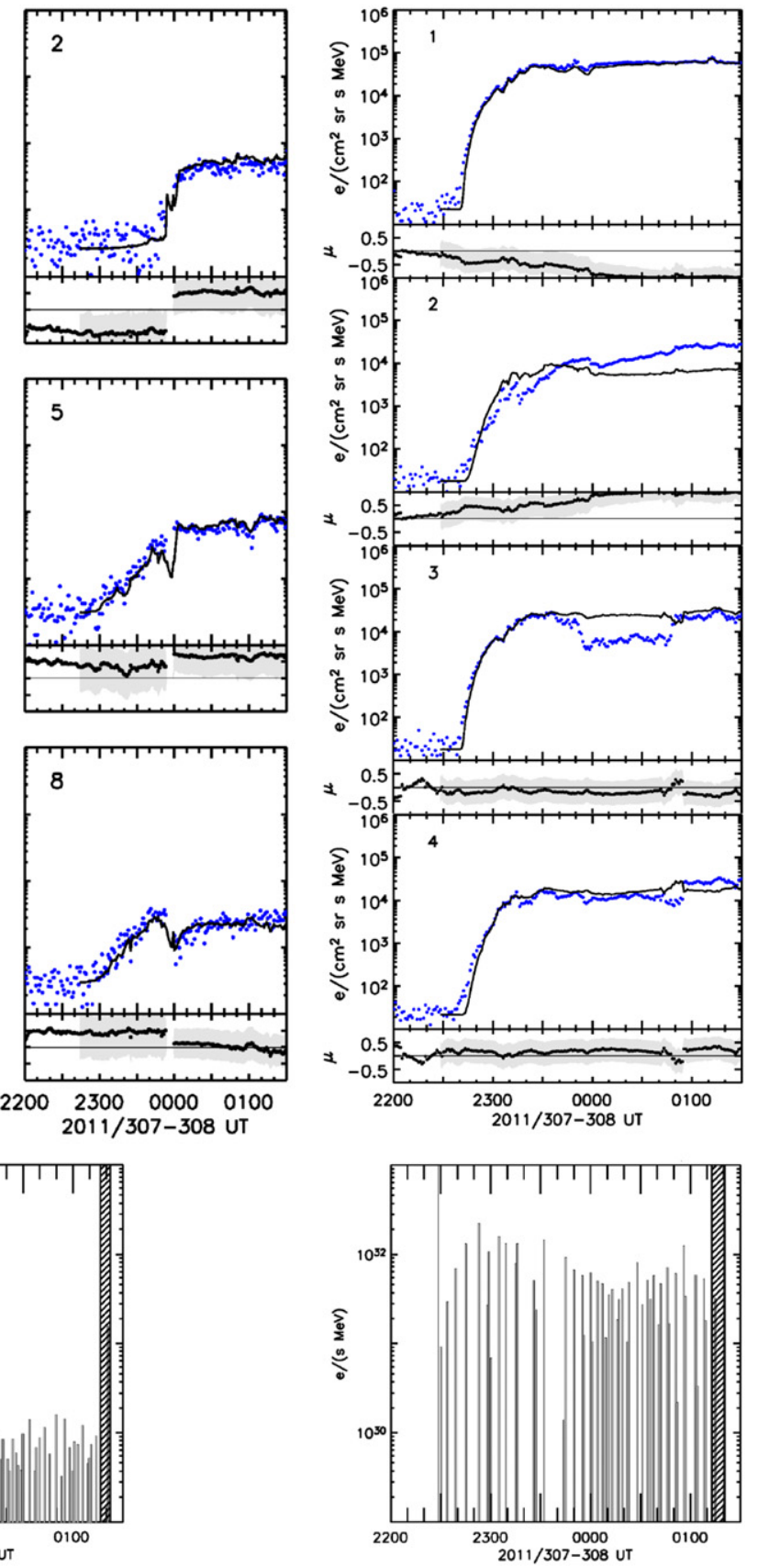

Figure 13. Monte Carlo transport model results for STEREO-B (left), ACE (center), and STEREO-A (right). The top panels show observed sector intensities (blue solid circles) compared with model predictions (solid black line). The small plots under each temporal profile shows the pitch-angle pointing for each sector. The three bottom panels show the injection profile at the Sun derived by the model best fits.

evidence that these particle increases observed over a broad longitudinal region have a common origin at a flaring region located at the distant solar backside (E152) from Earth's point of view. Several observational evidences support this common origin.

1. Very similar, strong, long-lasting type III radio-emission patterns were observed at the three locations close to $1 \mathrm{AU}$ (STEREO and Earth), showing high-frequency attenuation for STEREO-A and Wind, supporting the origin in a backside region from these two points of view.

2. Only one CME was observed during the period compatible with the particle release timing at the Sun. This moderately fast $\left(991 \mathrm{~km} \mathrm{~s}^{-1}\right)$ and wide CME was clearly originating from the backside flaring region.

3. Relativistic electrons and high-energy protons $(E \geqslant$ $100 \mathrm{MeV}$ ) were observed at the three locations (STEREO-A, STEREO-B, and Earth). While this is not extremely uncommon, it should be noted that not all SEP events show acceleration up to such high energies.

4. None of the four observation points revealed evidence of resolved multiple injections that might support acceleration at different sources closely spaced in time. The strong anisotropies observed at the onset of the event by the three $\mathrm{s} / \mathrm{c}$ close to $1 \mathrm{AU}$, indicate that scattering process 


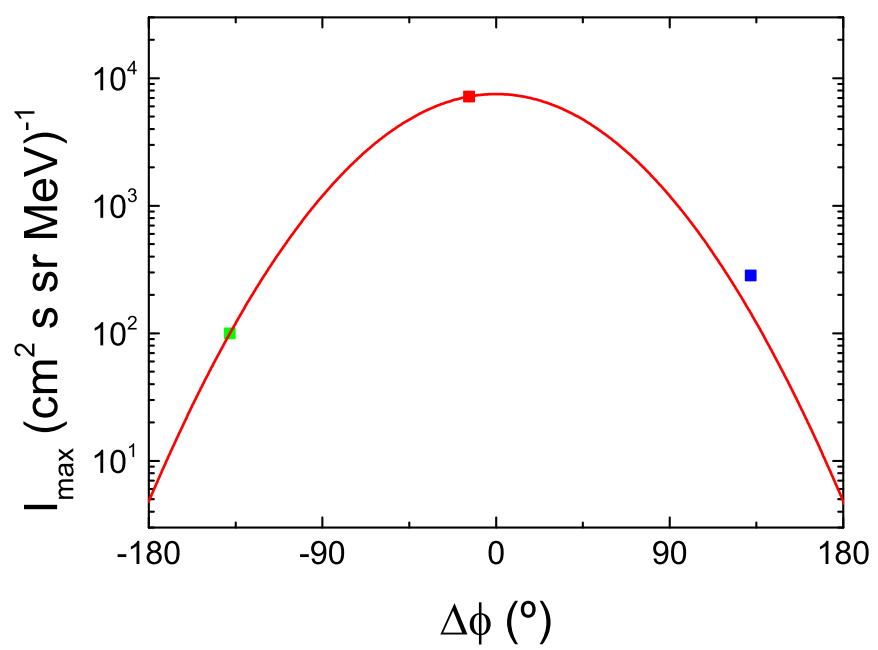

Figure 14. Longitudinal distribution of peak $235 \mathrm{keV}$ electron intensities observed by STEREO-A/SEPT (red), STEREO-B/SEPT (blue), and ACE/ EPAM-DE (green). The pre-event background has been subtracted and EPAM observations have been divided by an intercalibration factor of 1.3. The red line corresponds to a Gaussian fit with $\sigma=46.9 \pm 0.6$.

was not a factor to smooth the time-intensity profiles and blur individual separated injections.

5. Similar ${ }^{4} \mathrm{He} /{ }^{1} \mathrm{H}$ and $\mathrm{Fe} / \mathrm{O}$ ratios were observed by $\mathrm{SOHO} /$ $A C E$ and both STEREO spacecraft, characterized also by depleted ${ }^{4} \mathrm{He}$ abundance.

6. The longitudinal distribution of the electron peak intensities is well organized with respect to the longitudinal separation between the magnetic footpoints of each s/c and the backside flaring AR, with the highest intensity measured by the best connected s/c (STEREO-A). This behavior is consistent with previous multi-point observations of SEP events showing broad particle spread. Moreover, the independence of the distribution widths with the electron energy is a consequence of similar electron spectral shapes observed by both STEREO spacecraft and from ACE and $S O H O$. This energy-independent width can be represented by Gaussians with $\sigma \sim 46^{\circ}$. Similar values of $\sigma$ are found for protons.

The backside location of the single source implies that energetic particles during this period covered an extremely broad longitudinal spread in the inner heliosphere, filling a region of at least $258^{\circ}$ at $1 \mathrm{AU}$ (the longitudinal sector covered by STEREO$B$-Earth-STEREO-A). Considering the location of the nominal magnetic footpoints, particle injection close to the Sun should encompass a region at least $270^{\circ}$ wide. Indeed, the extrapolation of the Gaussian fits of the peak intensity as a function of the observer's footpoint separation (Figure 14) suggests that the SEP event would be detectable over the full $360^{\circ}$ longitudinal range at $1 \mathrm{AU}$.

From the analysis of the particle onsets times at different energies and from the modeling of the IP particle propagation, we conclude that the particle injection started at 22:29UT at the STEREO-A footpoint, well connected to the source region. This time is in good agreement with the type III and type II radio emission, and corresponds to a CME height of 2.3 solar radii. The injection at Earth and STEREO-B 's footpoints occurred shortly after, at 22:55 and 23:04 UT, respectively. This implies a relatively quick injection of the particles, before the CME reached 5.5 solar radii of heliocentric height, over a broad longitudinal span covering a region of at least $270^{\circ}$ close to the Sun. The fast longitudinal propagation and the clear anisotropies observed by STEREO-A, STEREO-B, and the nearEarth s/c supports the scenario of a direct particle injection at each observer's footpoint rather than cross-field diffusion in the interplanetary medium. The IP transport simulation results indicate extended injections lasting for several hours. The CME was accompanied by an EIT wave and by type II radio emission observed by both $S T E R E O$ spacecraft, indicating a shock propagating through the corona and the IP medium. However, radio and EUV observations did not reveal signatures of a possible source extending to the visible hemisphere from Earth's point of view. After the SEP event, MESSENGER and STEREO-B, located $45^{\circ}$ and $50^{\circ}$ far from the AR, consecutively observed the transit of an IP shock and an ICME almost certainly related to the November $3 \mathrm{CME}$. However, this ICME was observed neither by STEREO-A nor from Earth. Assuming that the ICME was symmetric and centered at the AR, this provides a lower limit of $100^{\circ}$ for the longitudinal full-width of the ICME and the IP shock. The lack of signatures at STEREO-A sets an upper limit of $204^{\circ}$ for the ICME and shock full-width. Therefore, while particle observations support the existence of a broad source when the CME was at a height of few solar radii, we did not find direct observational evidences of an exceptionally wide coronal or IP shock, large enough to explain the broad particle injection close to the Sun.

Prise et al. (2014) concluded that the CME lateral expansion was consistent with the particle release times at STEREO-A and STEREO-B footpoints, but was unable to explain the SEP onset at Earth. One possible hypothesis is that magnetic field lines below the source surface could shift Earth's magnetic connection by some tens of degrees westward from the nominal footpoint location, providing access to the region influenced by the CME lateral expansion. Our examination of the PFSS model results from GONG and the Solarsoft PFSS package suggests that the field lines below the source surface around Earth's nominal footpoint do not show such topology. An alternative explanation could be the presence of a previous ICME distorting the Parker spiral connecting to Earth. Only one ICME on November 7 17:00-23:00 UT was identified in $A C E$ in situ data that would be located between Sun and Earth at the time of particle onset.

\section{CONCLUSIONS}

Our observations show evidence that the circumsolar particle enhancement observed on 2011 November 3 is associated with activity originating at a single AR at the Sun. Clear anisotropies and short onset delays disfavor a major role of cross-field IP diffusion and support a quick particle spread close to the Sun, before the CME reached a heliocentric height of six solar radii. Particle timing requires that the source expands longitudinally at a rate of $\sim 5^{\circ}$ minute ${ }^{-1}$ (equivalent to $\sim 1000 \mathrm{~km} \mathrm{~s}^{-1}$ over the solar surface), reaching longitudes at least $138^{\circ}$ far from the AR. Neither remote sensing observations nor in situ ICME and IP shock measurements show visible signatures of a structure covering such wide extent. The markedly different signatures regarding timing and anisotropies of this event compared with the 2010 January 17 SEP event (in which IP cross-field diffusion likely played a major role; Dresing et al. 2012) support that the wide spread of SEPs in the heliosphere is caused by different physical processes with relative contributions that vary from event to event. 
The authors acknowledge the International Space Science Institute (ISSI) at Bern, Switzerland, for their funding of the team "Exploration of the inner Heliosphere-what we have learned from Helios and what we want to study with Solar Orbiter," led by Dr. W. Droege, and the useful discussions with the team members. R. G.-H. acknowledges the financial support by the Spanish Ministerio de Economía y Competitividad (projects AYA2011-29727-C02-01 and AYA2012-39810-C02-01). The STEREO/SEPT and SOHO/EPHIN projects are supported under grant 50 OC 0902 by the German Bundesministerium für Wirtschaft through the Deutsches Zentrum für Luft- und Raumfahrt (DLR). D. Lario acknowledges support from NASA under grant NNX11A083G. We acknowledge the different $A C E$, MESSENGER, SOHO, STEREO, and Wind instrument teams, and the STEREO and ACE Science Centers for providing the data used in this paper. MESSENGER data were downloaded from the Planetary Data System. This work utilizes data obtained by the Global Oscillation Network Group (GONG) program, managed by the National Solar Observatory, which is operated by AURA, Inc. under a cooperative agreement with the National Science Foundation. The data were acquired by instruments operated by the Big Bear Solar Observatory, High Altitude Observatory, Learmonth Solar Observatory, Udaipur Solar Observatory, Instituto de Astrofísica de Canarias, and Cerro Tololo InterAmerican Observatory.

\section{REFERENCES}

Acuña, M. H., Curtis, D., Scheifele, J. L., et al. 2008, SSRv, 136, 203 Agueda, N., Klein, K., Vilmer, N., et al. 2014, A\&A, 570, A5 Agueda, N., Vainio, R., Lario, D., \& Sanahuja, B. 2008, ApJ, 675, 1601 Agueda, N., Vainio, R., Lario, D., \& Sanahuja, B. 2010, A\&A, 519, A36 Anderson, B. J., Acuña, M. H., Lohr, D. A., et al. 2007, SSRv, 131, 417 Anderson, K. A., Sommers, J., Lin, R. P., et al. 1995, JGR, 100, 3 Andrews, G. B., Zurbuchen, T. H., Mauk, B. H., et al. 2007, SSRv, 131, 523 Aran, A., Sanahuja, B., \& Lario, D. 2008, AdSpR, 42, 1492

Aurass, H., Klein, K.-L., \& Mann, G. 1994, in Solar Dynamic Phenomena and Solar Wind Consequences, the Third SOHO Workshop, ed. J. J. Hunt (ESA SP-373; Noordwijk: ESA), 95

Bieber, J. W., Dröge, W., Evenson, P. A., et al. 2002, ApJ, 567, 622

Bothmer, V., Posner, A., Kunow, H., et al. 1997, in Correlated Phenomena at the Sun, in the Heliosphere and in Geospace, ed. A. Wilson (ESA SP-415; Noordwijk: ESA), 207

Bougeret, J. L., Goetz, K., Kaiser, M. L., et al. 2008, SSRv, 136, 487

Bougeret, J.-L., Kaiser, M. L., Kellogg, P. J., et al. 1995, SSRv, 71, 231

Brueckner, G. E., Howard, R. A., Koomen, M. J., et al. 1995, SoPh, 162, 357

Cane, H. V. 1988, JGR, 93, 1

Cane, H. V. 1995, NuPhS, 39, 35

Cane, H. V. 1996, in AIP Conf. Proc. 374, High Energy Solar Physics, ed. R. Ramaty, N. Mandzhavidze, \& X.-M. Hua (Melville, NY: AIP), 124 Cane, H. V. 2003, ApJ, 598, 1403

Cane, H. V., \& Erickson, W. C. 2003, JGRA, 108, 1203

Cane, H. V., Reames, D. V., \& von Rosenvinge, T. T. 1988, JGR, 93, 9555

Cane, H. V., \& Richardson, I. G. 2003, JGRA, 108, 1156

Chen, H., Ma, S., \& Zhang, J. 2013, ApJ, 778, 70

Chenette, D. L. 1980, JGR, 85, 2243

Chollet, E. E., Mewaldt, R. A., Cummings, A. C., et al. 2010, JGRA, 115, 12106 Cliver, E. W., Kahler, S. W., Neidig, D. F., et al. 1995, ICRC, 4, 257

Cliver, E. W., Thompson, B. J., Lawrence, G. R., et al. 2005, ICRC, 1, 121

Cohen, C. M. S., Mason, G. M., Mewaldt, R. A., \& Leske, R. A. 2012, in AIP Conf. Proc. 1500, Space Weather: The Space Radiation Environment, ed. Q. Hu, G. Li, G. P. Zank, X. Ao, O. Verkhoglyadova, \& J. H. Adams (Melville, NY: AIP), 74

da Costa, E., Jr., Tsurutani, B. T., Virgínia Alves, M., Echer, E., \& Lakhina, G. S. 2013, ApJ, 778, 180

Dalla, S., Balogh, A., Krucker, S., et al. 2003a, GeoRL, 30, 8035

Dalla, S., Balogh, A., Krucker, S., et al. 2003b, AnGeo, 21, 1367

Dalla, S., Marsh, M. S., Kelly, J., \& Laitinen, T. 2013, JGRA, 118, 5979

de Lucas, A., Schwenn, R., dal Lago, A., Marsch, E., \& Clúa de Gonzalez, A.

L. 2011, JASTP, 73, 1281

Desai, M. I., Mason, G. M., Gold, R. E., et al. 2006, ApJ, 649, 470
Dodson, H. W., \& Hedeman, E. R. 1969, SoPh, 9, 278

Dresing, N., Gómez-Herrero, R., Heber, B., et al. 2014, A\&A, 567, A27

Dresing, N., Gómez-Herrero, R., Klassen, A., et al. 2012, SoPh, 281, 281

Dröge, W., Kartavykh, Y. Y., Klecker, B., \& Kovaltsov, G. A. 2010, ApJ, 709, 912

Ergun, R. E., Larson, D., Lin, R. P., et al. 1998, ApJ, 503, 435

Ferreira, S. E. S., Potgieter, M. S., Burger, R. A., et al. 2001, JGR, 106, 29313

Galvin, A. B., Kistler, L. M., Popecki, M. A., et al. 2008, SSRv, 136, 437

Giacalone, J., \& Jokipii, J. R. 1999, ApJ, 520, 204

Giacalone, J., \& Jokipii, J. R. 2012, ApJL, 751, L33

Gold, R. E., Krimigis, S. M., Hawkins, S. E., III, et al. 1998, SSRv, 86, 541

Gómez-Herrero, R., Klassen, A., Heber, B., et al. 2007, in SOHO-17: 10 Years of SOHO and Beyond, ed. H. Lacoste \& L. Ouwehand (ESA SP-617; Noordwijk: ESA), 128

Gopalswamy, N., Xie, H., Yashiro, S., et al. 2012, SSRv, 171, 23

He, H.-Q., Qin, G., \& Zhang, M. 2011, ApJ, 734, 74

Heber, B., \& Potgieter, M. S. 2006, SSRv, 127, 117

Heras, A. M., Sanahuja, B., Lario, D., et al. 1995, ApJ, 445, 497

Heras, A. M., Sanahuja, B., Smith, Z. K., Detman, T., \& Dryer, M. 1992, ApJ, 391,359

Horne, R. B., Glauert, S. A., Meredith, N. P., et al. 2013, JSWSC, 3, A20

Hovestadt, D., Hilchenbach, M., Bürgi, A., et al. 1995, SoPh, 162, 441

Howard, R. A., Moses, J. D., Vourlidas, A., et al. 2008, SSRv, 136, 67

Huttunen-Heikinmaa, K., Valtonen, E., \& Laitinen, T. 2005, A\&A, 442, 673

Kahler, S. 1994, ApJ, 428, 837

Kahler, S., \& Ragot, B. R. 2006, ApJ, 646, 634

Kaiser, M. L. 2005, AdSpR, 36, 1483

Kallenrode, M.-B. 1993a, JGR, 98, 5573

Kallenrode, M.-B. 1993b, AdSpR, 13, 341

Kallenrode, M.-B. 2003, JPhG, 29, 965

Kallenrode, M.-B., \& Wibberenz, G. 1997, JGR, 102, 22311

Kallenrode, M.-B., Wibberenz, G., \& Hucke, S. 1992, ApJ, 394, 351

Kelly, J., Dalla, S., \& Laitinen, T. 2012, ApJ, 750, 47

Kienreich, I. W., Temmer, M., \& Veronig, A. M. 2009, ApJL, 703, L118

Klassen, A., Aurass, H., Mann, G., \& Thompson, B. J. 2000, A\&AS, 141, 357

Klassen, A., Gómez-Herrero, R., \& Heber, B. 2011, SoPh, 273, 413

Klassen, A., Gómez-Herrero, R., Heber, B., et al. 2012, A\&A, 542, A28

Klein, K.-L., Krucker, S., Lointier, G., \& Kerdraon, A. 2008, A\&A, 486, 589

Kocharov, L., Reiner, M. J., Klassen, A., Thompson, B. J., \& Valtonen, E. 2010, ApJ, 725, 2262

Krucker, S., Larson, D. E., Lin, R. P., \& Thompson, B. J. 1999, ApJ, 519, 864

Laitinen, T., Dalla, S., \& Marsh, M. S. 2013, ApJL, 773, L29

Lario, D., Aran, A., Gómez-Herrero, R., et al. 2013, ApJ, 767, 41

Lario, D., Kallenrode, M.-B., Decker, R. B., et al. 2006, ApJ, 653, 1531

Lario, D., Roelof, E. C., Decker, R. B., \& Reisenfeld, D. B. 2003, AdSpR, 32, 579

Lario, D., Sanahuja, B., \& Heras, A. M. 1998, ApJ, 509, 415

Lee, M. A. 2005, ApJS, 158, 38

Lemen, J. R., Title, A. M., Akin, D. J., et al. 2012, SoPh, 275, 17

Li, G., Moore, R., Mewaldt, R. A., Zhao, L., \& Labrador, A. W. 2012, SSRv, 171,141

Li, G., \& Zank, G. P. 2005, GeoRL, 32, 2101

Li, G., Zank, G. P., \& Rice, W. K. M. 2003, JGRA, 108, 1082

Li, G., Zank, G. P., \& Rice, W. K. M. 2005, JGRA, 110, 6104

Lin, R. P. 1990, in IAU Symp. 142, Basic Plasma Processes on the Sun, ed. E. R. Priest \& V. Krishan (Cambridge: Cambridge Univ. Press), 467

Lintunen, J., \& Vainio, R. 2004, A\&A, 420, 343

Luhmann, J. G., Curtis, D. W., Schroeder, P., et al. 2008, SSRv, 136, 117

Luhmann, J. G., Ledvina, S. A., Krauss-Varban, D., Odstrcil, D., \& Riley, P. 2007, AdSpR, 40, 295

Luhmann, J. G., Ledvina, S. A., Odstrcil, D., et al. 2010, AdSpR, 46, 1

Malandraki, O. E., Marsden, R. G., Lario, D., et al. 2009, ApJ, 704, 469

Malandraki, O. E., Sarris, E. T., Lanzerotti, L. J., et al. 2002, JASTP, 64, 517

Mann, G., Klassen, A., Aurass, H., \& Classen, H.-T. 2003, A\&A, 400, 329

Marsh, M. S., Dalla, S., Kelly, J., \& Laitinen, T. 2013, ApJ, 774, 4

Mason, G. M., Mazur, J. E., Dwyer, J. R., et al. 2004, ApJ, 606, 555

Masson, S., Aulanier, G., Pariat, E., \& Klein, K.-L. 2012, SoPh, 276, 199

Mazur, J. E., Mason, G. M., Dwyer, J. R., et al. 2000, ApJL, 532, L79

McComas, D. J., Bame, S. J., Barker, P., et al. 1998, SSRv, 86, 563

McKibben, R. B., Connell, J. J., Lopate, C., et al. 2003, AnGeo, 21, 1217

Mewaldt, R. A., Cohen, C. M. S., Cook, W. R., et al. 2008, SSRv, 136, 285

Mewaldt, R. A., Cohen, C. M. S., Mason, G. M., et al. 2013, in AIP Conf. Proc.

1539, Solar Wind 13, ed. G. P. Zank, J. Borovsky, R. Bruno, J. Cirtain, S.

Cranmer, H. Elliott, J. Giacalone, W. Gonzalez, G. Li, E. Marsch, E. Moebius,

N. Pogorelov, J. Spann, \& O. Verkhoglyadova (Melville, NY: AIP), 116

Miteva, R., Klein, K.-L., Kienreich, I., et al. 2014, SoPh, 289, 2601

Müller-Mellin, R., Böttcher, S., Falenski, J., et al. 2008, SSRv, 136, 363 
Müller-Mellin, R., Kunow, H., Fleißner, V., et al. 1995, SoPh, 162, 483

Nelson, G. J., \& Robinson, R. D. 1975, PASAu, 2, 370

Newkirk, G., Jr., \& Wentzel, D. G. 1978, JGR, 83, 2009

Ng, C. K., Reames, D. V., \& Tylka, A. J. 2003, ApJ, 591, 461

Nitta, N. V., Aschwanden, M. J., Boerner, P. F., et al. 2013a, SoPh, 288, 241

Nitta, N. V., \& De Rosa, M. L. 2008, ApJL, 673, L207

Nitta, N. V., Schrijver, C. J., Title, A. M., \& Liu, W. 2013b, ApJ, 776, 58

Palmer, I. D. 1982, RvGSP, 20, 335

Park, J., Innes, D. E., Bucik, R., \& Moon, Y.-J. 2013, ApJ, 779, 184

Patsourakos, S., \& Vourlidas, A. 2012, SoPh, 281, 187

Prise, A., Harra, L., Matthews, S., Long, D., \& Aylward, A. 2014, SoPh, 289, 1731

Reames, D. V. 1988, ApJL, 330, L71

Reames, D. V. 1999, SSRv, 90, 413

Reames, D. V. 2009a, ApJ, 693, 812

Reames, D. V. 2009b, ApJ, 706, 844

Reames, D. V. 2013, SSRv, 175, 53

Reames, D. V., \& Ng, C. K. 2002, ApJL, 577, L59

Reid, G. C. 1964, JGR, 69, 2659

Reinhard, R., \& Wibberenz, G. 1974, SoPh, 36, 473

Richardson, I. G., \& Cane, H. V. 1993, JGR, 98, 15295

Richardson, I. G., \& Cane, H. V. 1996, JGR, 101, 27521

Richardson, I. G., Cane, H. V., \& von Rosenvinge, T. T. 1991, JGR, 96, 7853

Richardson, I. G., von Rosenvinge, T. T., Cane, H. V., et al. 2014, SoPh, 289, 3059

Roelof, E. C. 2008, in AIP Conf. Proc. 1039, Particle Acceleration and Transport in the Heliosphere and Beyond, ed. G. Li, Q. Hu, O. Verkhoglyadova, G. P. Zank, R. P. Lin, \& J. Luhmann (Melville, NY: AIP), 174

Rouillard, A. P., Sheeley, N. R., Tylka, A., et al. 2012, ApJ, 752, 44
Ruffolo, D., Khumlumlert, T., \& Youngdee, W. 1998, JGR, 103, 20591

Sáiz, A., Evenson, P., Ruffolo, D., \& Bieber, J. W. 2005, ApJ, 626, 1131

Schrijver, C. J., \& De Rosa, M. L. 2003, SoPh, 212, 165

Schrijver, C. J., \& Title, A. M. 2011, JGRA, 116, 4108

Schrijver, C. J., Title, A. M., Yeates, A. R., \& DeRosa, M. L. 2013, ApJ, 773,93

Shen, Y., Liu, Y., \& Su, J. 2012, ApJ, 750, 12

Smith, C. W., L'Heureux, J., Ness, N. F., et al. 1998, SSRv, 86, 613

Stone, E. C., Cohen, C. M. S., Cook, W. R., et al. 1998, SSRv, 86, 357

Tan, L. C., Reames, D. V., Ng, C. K., Saloniemi, O., \& Wang, L. 2009, ApJ, 701,1753

Thejappa, G., MacDowall, R. J., \& Bergamo, M. 2012, JGRA, 117, 8111

Thompson, B. J., \& Myers, D. C. 2009, ApJS, 183, 225

Torsti, J., Kocharov, L., Teittinen, M., et al. 1999, JGR, 104, 9903

Torsti, J., Riihonen, E., \& Kocharov, L. 2004, ApJL, 600, L83

Torsti, J., Valtonen, E., Lumme, M., et al. 1995, SoPh, 162, 505

Vainio, R., Valtonen, E., Heber, B., et al. 2013, JSWSC, 3, A12

Verkhoglyadova, O. P., Li, G., Ao, X., \& Zank, G. P. 2012, ApJ, 757, 75

Verkhoglyadova, O. P., Li, G., Zank, G. P., Hu, Q., \& Mewaldt, R. A. 2009, ApJ, 693,894

Verkhoglyadova, O. P., Li, G., Zank, G. P., et al. 2010, JGRA, 115, 12103

von Rosenvinge, T. T., Reames, D. V., Baker, R., et al. 2008, SSRv, 136, 391

Wang, H., Chae, J., Yurchyshyn, V., et al. 2001, ApJ, 559, 1171

Wang, Y., Qin, G., \& Zhang, M. 2012, ApJ, 752, 37

Wibberenz, G., \& Cane, H. V. 2006, ApJ, 650, 1199

Wiedenbeck, M. E., Mason, G. M., Cohen, C. M. S., et al. 2013, ApJ, 762,54

Zank, G. P., Li, G., \& Verkhoglyadova, O. 2007, SSRv, 130, 255

Zank, G. P., Rice, W. K. M., \& Wu, C. C. 2000, JGR, 105, 25079 